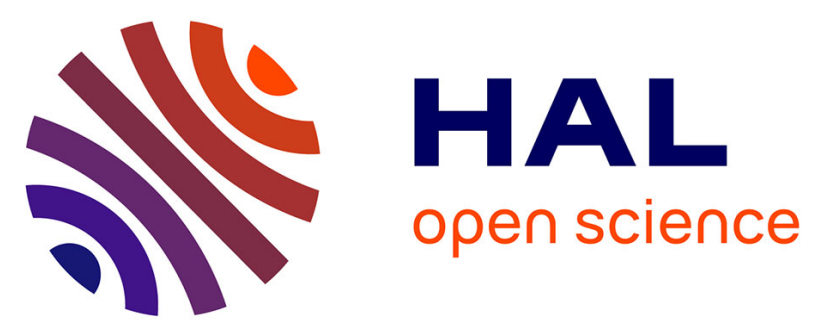

\title{
Tuning Sizes, Morphologies, and Magnetic Properties of Monocore Versus Multicore Iron Oxide Nanoparticles through the Controlled Addition of Water in the Polyol Synthesis
}

Gauvin Hémery, Anthony C. Keyes Jr, Eneko Garaio, Irati Rodrigo, Jose Angel Garcia, Fernando Plazaola, Elisabeth C Garanger, Olivier Sandre

\section{To cite this version:}

Gauvin Hémery, Anthony C. Keyes Jr, Eneko Garaio, Irati Rodrigo, Jose Angel Garcia, et al.. Tuning Sizes, Morphologies, and Magnetic Properties of Monocore Versus Multicore Iron Oxide Nanoparticles through the Controlled Addition of Water in the Polyol Synthesis. Inorganic Chemistry, 2017, 56 (14), pp.8232-8243. 10.1021/acs.inorgchem.7b00956 . hal-01567664v3

\section{HAL Id: hal-01567664 \\ https://hal.science/hal-01567664v3}

Submitted on 17 Nov 2019

HAL is a multi-disciplinary open access archive for the deposit and dissemination of scientific research documents, whether they are published or not. The documents may come from teaching and research institutions in France or abroad, or from public or private research centers.
L'archive ouverte pluridisciplinaire $\mathbf{H A L}$, est destinée au dépôt et à la diffusion de documents scientifiques de niveau recherche, publiés ou non, émanant des établissements d'enseignement et de recherche français ou étrangers, des laboratoires publics ou privés.

\section{(ㅇ)(1) $\$$}

Distributed under a Creative Commons Attribution - NonCommercial - NoDerivatives $\mid 4.0$ 


\section{Tuning Sizes, Morphologies, and Magnetic}

\section{Properties of Monocore Versus Multicore Iron}

\section{Oxide Nanoparticles through the Controlled}

\section{Addition of Water in the Polyol Synthesis}

$\underline{\text { Gauvin Hemery }}^{a}, \underline{\text { Anthony C. Keyes Jr. }}^{a}, \underline{\text { Eneko Garaio }}^{b} * \underline{\text { Irati Rodrigo }}^{b, c}$, Jose Angel Garcia ${ }^{c, d}$, $\underline{\text { Fernando Plazaola }}^{b}$, Elisabeth Garanger $^{a}$, Olivier Sandre $^{a *}$

${ }^{a}$ LCPO, CNRS UMR 5629/ Univ. Bordeaux/ Bordeaux-INP, ENSCBP 16 avenue Pey Berland, 33607 Pessac, France

${ }^{\mathrm{b}}$ Elektrizitatea eta Elektronika Saila, UPV/EHU, 48940 Leioa, Spain

${ }^{\mathrm{c}}$ BCMaterials, Parque Tecnológico de Bizkaia, Ed. 50, 48160 Derio, Spain

${ }^{\mathrm{d}}$ Fisika Aplikatua II Saila, UPV/EHU, 48940 Leioa, Spain

KEYWORDS Polyol synthesis, iron oxide, nanoparticles, magnetic hyperthermia, MRI contrast agent, nanoflowers, multi-core, mono-core

ABSTRACT The polyol route is a versatile and up-scalable method to produce large batches of iron oxide nanoparticles with well-defined structures and magnetic properties. Importance of 
This document is the author manuscript version of published paper Inorganic Chemistry, 2017, 56(14), 8232-8243, after peer review and technical editing by the publisher. Final version visible on DOI:10.1021/acs.inorgchem.7b00956

parameters such as temperature and reaction time, heating profile, nature of polyol solvent or of organometallic precursors on nanostructure and properties has already been described in the literature. Yet, the crucial role of water in the forced hydrolysis pathway has never been reported despite its mandatory presence for nanoparticle production. This communication investigates the influence of the water amount and temperature at which it is injected in the reflux system for either a pure polyol solvent system or a mixture with poly(hydroxy)amine. Distinct morphologies of nanoparticles were thereby obtained, from ultra-ultra-small smooth spheres down to $4 \mathrm{~nm}$ in diameter to larger ones up to $37 \mathrm{~nm}$. Well-defined multi-core assemblies with narrow grain size dispersity termed nanoflowers were also synthesized. A diverse and large library of samples was obtained by playing with the nature of solvents and amount of added water while keeping all other parameters constant. The different morphologies lead to magnetic nanoparticles suitable for important biomedical applications such as magnetic hyperthermia, MRI contrast agent, or both.

\section{INTRODUCTION}

Magnetic iron oxide nanoparticles (IONPs) find applications in the biomedical field as diagnostic tools and innovative therapies as they provide contrasting properties in magnetic resonance imaging (MRI) and also serve as heat mediators in magnetic fluid hyperthermia (MFH) and for triggered drug delivery. ${ }^{1}$ As IONPs are biocompatible, they are among the best candidates over alternative magnetic nanomaterials for health applications. Various synthetic pathways have been explored for their production, ${ }^{2}$ the most common ones being the alkaline coprecipitation, ${ }^{3}$ the polyol process, ${ }^{4}$ the thermal degradation of organometallic precursors, ${ }^{5}$ and the hydrothermal treatment. ${ }^{6}$ The polyol route is an interesting compromise when taking into account the ease of synthesis, scalability, cost-efficiency, and control over the morphology. This 
This document is the author manuscript version of published paper Inorganic Chemistry, 2017, 56(14), 8232-8243, after peer review and technical editing by the publisher. Final version visible on DOI:10.1021/acs.inorgchem.7b00956

process leads to nanoparticles with morphologies ranging from smooth spheres to more complex structures such as the so-called "nanoflowers". Other strategies were reported to directly produce flower-like structures in water such as the co-precipitation performed in presence of excess polysaccharide, $^{7}$ or in a microwave reactor. ${ }^{8}$ The polyol synthesis of multi-core IONPs was introduced by Caruntu et al. ${ }^{4}$ In this reaction pathway, the solvent acts simultaneously as a complexing agent for iron chloride precursors and as high boiling point solvent, with reflux temperatures usually in the order of $220{ }^{\circ} \mathrm{C}$. Nanoflowers are to date amongst the best IONPs in terms of efficiency for heating under an applied alternating magnetic field (AMF) and as negative (transverse $T_{2}$ ) MRI contrast agents, as previously reported. ${ }^{9}$ At equivalent concentrations of iron, the outstanding heating properties of these assemblies of small grains into larger raspberry-like structures were related to a frustrated super-spin glass state. ${ }^{10}$ Regarding mechanistic pathway of the synthesis, tentative descriptions of the intermediary states of reaction were proposed, ${ }^{11}$ together with the role of the polyol solvent in orienting the morphology. ${ }^{12}$ Different reaction conditions were studied in the literature, with variable parameters such as the choice of the polyol solvent, ${ }^{12}$ temperature and pressure, ${ }^{13}$ reaction time and heating ramp slope, ${ }^{14}$ alkaline $\mathrm{pH}^{15}$ and presence of adsorbed capping agents. ${ }^{16}$ The main applications envisioned for nanoflowers are as nanoheaters for MFH and efficient negative $\left(T_{2}\right)$ contrast agents for MRI, as ascribed to their large magnetic moment and large intrinsic magnetization $M_{\mathrm{d}}$ (magnetic mono-domain moment divided by the particle volume). The main challenge to overcome for utilizing these superior magnetic properties in biological media is to prevent particle aggregation that can be evidenced by a non-reversible magnetization curve in static magnetic field. With large magnetic moments, nanoflowers experiment strong magnetic dipolar interactions. Moreover, the coating of their surface by a residual organic layer can render them 
This document is the author manuscript version of published paper Inorganic Chemistry, 2017, 56(14), 8232-8243, after peer review and technical editing by the publisher. Final version visible on DOI:10.1021/acs.inorgchem.7b00956

difficult to peptize as stable aqueous colloidal suspensions, especially in physiological media. However, we found that all nanoparticles synthesized in our study, even the largest nanoflowers, show superparamagnetic behavior at room temperature with no hysteresis of their magnetization curve under DC field at room temperature, which is a prerequisite for their use in biological applications (yet still necessitating further coating to reach stealthiness in blood circulation).

In the course of our studies, we evidenced the crucial role of water in the polyol synthesis of IONPs. We report thereafter an extensive study describing how the amount and way of adding water in the synthesis influence the final nanoparticle (NP) morphology. A library of waterdispersible IONPs was therefore successfully synthesized, with tunable diameters from $\sim 4$ to $\sim 37$ $\mathrm{nm}$ and superior magnetic properties for MFH and MRI.

\section{EXPERIMENTAL SECTION}

\section{Materials}

Nitric acid $\left(\mathrm{HNO}_{3}, 70 \%\right)$ was from Fisher, ethyl acetate $(>99.5 \%)$ was from Sigma Aldrich, acetone (technical grade), ethanol (96\%), and diethyl ether $(100 \%)$ were from VWR. $N$ methyldiethanolamine (NMDEA, 99\%) was from Acros Organics, diethylene glycol (DEG, 99\%), sodium hydroxide microprills $(\mathrm{NaOH}, 98 \%)$, iron(III) nitrate nonahydrate $\left(\mathrm{Fe}\left(\mathrm{NO}_{3}\right)_{3} \cdot 9 \mathrm{H}_{2} \mathrm{O},>98 \%\right)$, and iron(II) chloride tetrahydrate $\left(\mathrm{FeCl}_{2} \cdot 4 \mathrm{H}_{2} \mathrm{O}, 98 \%\right)$ were from Alfa Aesar. Iron(III) chloride hexahydrate $\left(\mathrm{FeCl}_{3} \cdot 6 \mathrm{H}_{2} \mathrm{O},>97 \%\right)$ was from Panreac.

\section{Synthesis of iron oxide nanoparticles}

Nanoparticles were produced by adapting reaction conditions reported by Caruntu et al.: ${ }^{4} 80$ $\mathrm{mL}$ of either pure DEG or a mixture of DEG and NMDEA with volume ratios 1:1 v/v were 
This document is the author manuscript version of published paper Inorganic Chemistry, 2017, 56(14), 8232-8243, after peer review and technical editing by the publisher. Final version visible on DOI:10.1021/acs.inorgchem.7b00956

introduced in a three-neck round bottom flask flushed with nitrogen and stirred with a magnetic stir bar for one hour under inert atmosphere. $1.08 \mathrm{~g}(4 \mathrm{mmol})$ of $\mathrm{FeCl}_{3} \cdot 6 \mathrm{H}_{2} \mathrm{O}$ and $0.40 \mathrm{~g}(2$ mmol) of $\mathrm{FeCl}_{2} \cdot 4 \mathrm{H}_{2} \mathrm{O}$ were then dissolved overnight. In the meantime, $0.64 \mathrm{~g}(16 \mathrm{mmol})$ of $\mathrm{NaOH}$ was dissolved under magnetic stirring in $40 \mathrm{~mL}$ of either pure DEG or a 1:1 v/v mixture of DEG and NMDEA in a separate three-neck round bottom flask. The $\mathrm{NaOH}$ solution was flushed by bubbling nitrogen for one hour before mixing with the mixed iron(II,III) chloride solution. The color quickly turned from yellow to deep green. The mixture was then heated up to $220{ }^{\circ} \mathrm{C}$ (temperature ramp in around $30 \mathrm{~min}$ ) with an electronically controlled Digi-Mantle $\mathrm{TM}^{\mathrm{TM}}$ heating mantle (OMCA0250, Electrothermal $\left.{ }^{\mathrm{TM}}\right)$ set at full power, before letting the reaction to occur for a determined period of time, either with or without agitation at $500 \mathrm{rpm}$ by a mechanical stirring Teflon shaft inserted through the condenser aperture. Nanoparticles were then separated over a strong permanent ferrite magnet $\left(152 \times 101 \times 25.4 \mathrm{~mm}^{3}\right.$, Calamit Magneti ${ }^{\mathrm{TM}}$, Milano-Barcelona-Paris), washed three times with a mixture of ethanol and ethyl acetate (1:1 $\mathrm{v} / \mathrm{v}$ ), once with $10 \%$ nitric acid, twice with acetone, and twice with diethyl ether. NPs were then readily redispersed in water by stirring in open air to remove volatile solvents. At this stage, a black monophasic dispersion of IONPs was obtained. $8.6 \mathrm{~g}$ of iron(III) nitrate was then added as a strong oxidant by heating at $80{ }^{\circ} \mathrm{C}$ for $45 \mathrm{~min}$ while mechanically stirring. ${ }^{17}$ The colloidal solution then turned from clear black to clear brown-orange. The IONPs were flocculated by addition of $10 \%$ nitric acid and finally washed twice with acetone and twice again with diethyl ether. At this stage a deep orange-black dispersion of IONPs was obtained. The fluid was attracted by permanent magnets while staying in a single liquid phase, confirming that a true "ferrofluid" was obtained. 
This document is the author manuscript version of published paper Inorganic Chemistry, 2017, 56(14), 8232-8243, after peer review and technical editing by the publisher. Final version visible on DOI:10.1021/acs.inorgchem.7b00956

\section{Sample nomenclature}

Each final product is designated according to the main synthesis parameters, i.e. the solvent $(D$ for pure DEG, $N$ for DEG/NMDEA 1:1), the volume of water in $\mu \mathrm{L}$ added to $120 \mathrm{~mL}$ of solvent (the subscript $H I$ or $H U$ being added to specify if water was injected to the reaction mixture at solvent reflux - hot injection - or by heating-up from room temperature, respectively), and the reaction time. For instance, the sample name $N 500_{\mathrm{HU}^{-}} 5 h$ indicates that $500 \mu \mathrm{L}$ of $\mathrm{H}_{2} \mathrm{O}$ were added to $120 \mathrm{~mL}$ DEG/NMDEA (1:1) and the reaction mixture was heated up to reflux for 5 hours. The sample name $D 5000_{\mathrm{HI}^{-}} 2 \mathrm{~m}$ indicates that $5,000 \mu \mathrm{L}$ of $\mathrm{H}_{2} \mathrm{O}$ were added through a septum to $120 \mathrm{~mL}$ of iron(II,III) precursors in boiling DEG, then let for $20 \mathrm{~min}$ before cooling).

Table 1: Batch names and their corresponding conditions of synthesis. Asterisk designates a reaction performed under 'natural mixing' i.e. by diffusion and convection yet no stirring.

\begin{tabular}{|c|c|}
\hline Batch name & Nomenclature \\
\hline $15 \mathrm{ff}$ & $N 1000_{H^{-}}-5 h$ \\
\hline $17 \mathrm{ff}$ & $\mathrm{D} 5000_{H I}-20 \mathrm{~m}$ \\
\hline $25 \mathrm{ff}$ & $N 500_{H U^{-}} 4 h$ \\
\hline $30 \mathrm{ff}$ & $N 1000_{H U^{-}} 5 h$ \\
\hline $31 \mathrm{ff}$ & $N 500_{H U^{-}} 1 h$ \\
\hline $32 \mathrm{ff}$ & $N 500_{H U^{-}} 5 h$ \\
\hline $34 \mathrm{ff}$ & $N 100_{H U^{-}} 5 h^{*}$ \\
\hline $35 \mathrm{ff}$ & $N 100_{H U^{-}} 5 h$ \\
\hline $36 \mathrm{ff}$ & $N 100_{H U}-5 h$ \\
\hline
\end{tabular}

Transmission Electron Microscopy (TEM) 
TEM was performed on a Hitachi ${ }^{\mathrm{TM}} \mathrm{H} 7650$ microscope with an acceleration voltage of $80 \mathrm{kV}$. TEM images were acquired with an ORIUS $^{\mathrm{TM}}$ SC1000 large format (11 MPx) camera. Samples were prepared by nebulizing NP dispersions at $1 \mathrm{~g} \cdot \mathrm{L}^{-1}$ concentration on Formvar ${ }^{\mathrm{TM}}$ carbon-coated 200 mesh copper grids from Agar Scientific ${ }^{\mathrm{TM}}$ and leaving them to dry at room temperature. NP size distributions were obtained by measuring more than $100 \mathrm{NPs}$ with the ImageJ freeware (https://imagej.nih.gov/ij/). Size-histograms were fitted to a log-normal distribution law of diameters $P(d)$ with optimized values of median diameter $\alpha$ and non-dimensioned width $\beta$ :

$$
P(d)=\frac{1}{d \cdot \beta \sqrt{2 \pi}} \cdot \exp \left(\frac{-(\ln (d)-\ln (\alpha))^{2}}{2 \beta^{2}}\right)
$$

In addition, the mean sizes $d_{0}=<d>$ (number-averages) and standard deviations $\sigma=<(d$ $<d>)^{2}>^{1 / 2}$ were calculated using the classical Gaussian statistics formulas.

\section{Dynamic Light Scattering (DLS) and Zetametry}

A Nanosizer $^{\mathrm{TM}}$ Nano ZS90 from Malvern ${ }^{\mathrm{TM}}$, UK, was used to measure $\zeta$ potentials, $Z$-average hydrodynamic diameters $\left(D_{\mathrm{h}}\right)$, and polydispersity indexes (PDI). The $2^{\text {nd }}$ order Cumulant fit was used for analysing DLS data into a size distribution (the PDI being defined as the ratio of the $2^{\text {nd }}$ order coefficient to the square of the $1^{\text {st }}$ order one in the series ${ }^{18}$ ). The Smoluchowski equation was used to convert the measured electrophoretic mobility into a $\zeta$ potential value in $\mathrm{mV}$.

\section{Attenuated total reflection infrared spectroscopy}

ATR-IR spectroscopy was carried on a GladiATR ${ }^{\mathrm{TM}}$ device from Pike Technologies mounted on a Bruker Vertex ${ }^{\mathrm{TM}} 70$ FT-IR spectrometer. Typically a drop of sample was dried on the 
This document is the author manuscript version of published paper Inorganic Chemistry, 2017, 56(14), 8232-8243, after peer review and technical editing by the publisher. Final version visible on DOI:10.1021/acs.inorgchem.7b00956

diamond prism using a hair blower followed by a 64 scans measurement. Spectra were analyzed with the Opus ${ }^{\mathrm{TM}}$ software.

\section{Proton relaxometry}

Samples were prepared at concentrations of $6 \mathrm{mM}_{\mathrm{Fe}}$. NMR tubes (7.5 mm outer diameter) were filled with $1 \mathrm{~mL}$ of each sample, and inserted in a Bruker ${ }^{\mathrm{TM}} \mathrm{mq} 60$ relaxometer equipped with a $60 \mathrm{MHz} / 1.41$ Tesla magnet. The samples were left to thermalize to $37{ }^{\circ} \mathrm{C}$ using a Julabo ${ }^{\mathrm{TM}} \mathrm{f} 25$ ED circulation bath. Following recommended protocols in proton relaxometry, ${ }^{19}$ longitudinal $T_{1}$ relaxation times were measured using an inversion-recovery sequence of first duration of $\sim 0.1 \times T_{1}$ and final duration of $\sim 3 \times T_{1}$ with a recycling delay (RD) of $\sim 5 \times T_{1}$ between two of the 4 acquisitions, 10 data points per scan, and an automatic RF receiver gain. Transverse $T_{2}$ relaxation times were measured using Car-Purcell-Meiboom-Gill (CPMG) sequence, with delay time $\tau$ of $0.04 \mathrm{~ms}$ between the $90^{\circ}$ rotation to transverse plane and the $180^{\circ}$ focusing pulse, a duration time of $3 \times T_{2}, \mathrm{RD}$ of $5 \times T_{1}$, and automatic receiver gain. The number of acquisition points was set by dividing the duration time by the delay time $\tau$.

\section{Magnetic fluid hyperthermia}

NPs were dispersed at concentrations of $3 \mathrm{~g} \cdot \mathrm{L}^{-1}$ in diluted $\mathrm{HNO}_{3}$ (at $\mathrm{pH} 2$ ) to preserve their colloidal stability. The samples were placed in $500 \mu \mathrm{L}$ plastic cuvettes, whose caps were pierced with a needle to introduce a fiber optics temperature probe of $420 \mu \mathrm{m}$ outer diameter (medical range OTG-M420 fiber, Opsens ${ }^{\mathrm{TM}}$, Québec, QC, Canada) and measure temperature profiles versus time. Samples were thermalized at $37{ }^{\circ} \mathrm{C}$ using a glass-water jacket connected to a temperature bath until reaching equilibrium. The heat generation by magnetic NPs was triggered 
This document is the author manuscript version of published paper Inorganic Chemistry, 2017, 56(14), 8232-8243, after peer review and technical editing by the publisher. Final version visible on DOI:10.1021/acs.inorgchem.7b00956

using an induction coil (4-turn of $3.5 \mathrm{~mm}$ diameter hollow - $0.4 \mathrm{~mm}$ wall - copper tubing, 55 $\mathrm{mm}$ outer diameter, $48 \mathrm{~mm}$ inner diameter, $34.5 \mathrm{~mm}$ height) fed by a Minimax Junior ${ }^{\mathrm{TM}}$ 1TS 3.5 kW generator (Seit Elettronica ${ }^{\mathrm{TM}}$, Italy) applying an alternating magnetic field (AMF) at maximum amplitude $H_{\text {app }}$ of $10.2 \mathrm{kA} \cdot \mathrm{m}^{-1}$ and at a frequency $f$ of $755 \mathrm{kHz}$ as determined by finite element modelling. ${ }^{20}$ The amplitude and frequency of the magnetic field were corroborated by measuring the electromotive force in a scout coil (made of a single turn of $17.5 \mathrm{~mm}$ diameter) and an oscilloscope (Agilent $\left.{ }^{\mathrm{TM}} 54641 \mathrm{~A}\right)$. The AMF was applied for 5 min while recording the elevation of temperature and measuring its slope at early times (within first $5 \mathrm{~s}$ ).

\section{Static (DC) magnetization}

DC magnetization curves of the NP aqueous dispersions were obtained up to 1.79 Tesla on a homemade vibrating sample magnetometer (VSM) at the SGIker facility (UPV/EHU). The magnetic field was measured by a gaussmeter whereas the signal was conditioned by a Stanford $^{\mathrm{TM}}$ SR810DSP lock-in amplifier controlled by a PC under a LabVIEW ${ }^{\mathrm{TM}}$ program. All VSM measurements were performed at room temperature, the applied field $H_{\text {app }}$ being cycled from $1.43 \times 10^{6} \mathrm{~A} \cdot \mathrm{m}^{-1}$ to $-1.43 \times 10^{6} \mathrm{~A} \cdot \mathrm{m}^{-1}$ and then back to $1.43 \times 10^{6} \mathrm{~A} \cdot \mathrm{m}^{-1}$.

\section{Zero field cooling - field cooling (ZFC-FC) magnetometry}

ZFC-FC experiments were conducted on a magnetic property measurement system (MPMS ${ }^{\mathrm{TM}}$ 7T from Quantum Design ${ }^{\mathrm{TM}}$, San Diego, CA, USA). This ultrasensitive magnetometer was previously calibrated by $\mathrm{Y}_{3} \mathrm{Fe}_{5} \mathrm{O}_{12}$ garnet $1 \mathrm{~mm}$ diameter sphere (standard reference materials 2853) and reset after each measurement. Estimates of the blocking temperature were made according to a previously published protocol. ${ }^{21,22}$ 


\section{Equivalent iron titration in IONP suspensions}

The equivalent iron molarity [Fe] was measured by a disruptive photometric assay, using the characteristic absorption peak at $350 \mathrm{~nm}$ of the $\left[\mathrm{Fe}(\mathrm{Cl})_{6}\right]^{3-}$ complex when an aliquot of the suspension was dissolved in concentrated hydrochloric acid ( $\mathrm{HCl} 5 \mathrm{M})$, according to previous calibration law $\mathrm{OD}_{350 \mathrm{~nm}, 2 \mathrm{~mm}}=0.5043 \times[\mathrm{Fe}]_{\mathrm{mM}}+0.0172$. Then $[\mathrm{Fe}]$ was converted into iron oxide weight assuming pure $\gamma-\mathrm{Fe}_{2} \mathrm{O}_{3}$ composition ( $~ 80 \mathrm{~g}$ per mol of iron).

\section{Small angle neutron scattering (SANS)}

SANS curves were acquired on the PACE spectrometer of the LLB-CEA Saclay, France, equipped with an isotropic $\mathrm{BF}_{3}$ detector made of 30 concentric rings of $1 \mathrm{~cm}$ width each. Three configurations were used to cover overlapping $q$ ranges of $3.1 \times 10^{-3}-3.3 \times 10^{-2}, 1.4 \times 10^{-2}-$ $1.5 \times 10^{-1}$, and $4.7 \times 10^{-2}-0.48 \AA^{-1}$, with the following values of sample-to-detector distance $D$ and neutron wavelength $\lambda: D=4.57 \mathrm{~m}$ and $\lambda=13 \AA, D=2.85 \mathrm{~m}$ and $\lambda=4.6 \AA, D=0.87 \mathrm{~m}$ and $\lambda=4.6 \AA$. After dividing scattered intensity by the transmission factor, subtracting the incoherent background, and normalizing by the flat signal of a cuvette filled with light water to correct the detector efficiency, the absolute intensity in $\mathrm{cm}^{-1}$ was obtained. ${ }^{23}$ Curve fitting by a polydisperse sphere form factor gave the $R_{\mathrm{SANS}}$ radius and its standard deviation, and Porod's law at high $q$ knowing volume fraction $\phi$ gave an estimate of the specific area $A_{\text {spe }}$ (Figure S1).

\section{RESULTS AND DISCUSSIONS}

In the course of our work, we postulated that water traces are incorporated in the highly hygroscopic polyol solvent and that this initial water content in the mixture before reaction is a 
This document is the author manuscript version of published paper Inorganic Chemistry, 2017, 56(14), 8232-8243, after peer review and technical editing by the publisher. Final version visible on DOI:10.1021/acs.inorgchem.7b00956

key parameter to produce well-defined magnetic IONPs. This led us to carry out an extensive study on the role of controlled additions of water in the synthesis of iron oxide nanoparticles in a mixture of polyols (DEG, NMDEA). Different batches of IONPs were thereby produced by varying reaction parameters such as reaction time, solvent system, amount and timing of injections of water amounts, i.e. hot injection at solvent reflux vs. heating-up of the reaction mixture including water. Since the two major biomedical applications of IONPs are for MFH and as MRI contrast agents, we have dedicated our efforts to produce well-defined IONPs suitable for these two applications. Large (several tens of $\mathrm{nm}$ ) IONPs were produced for MFH, while ultra-ultra-small superparamagnetic iron oxide NPs (UUSPIO) of just a few nm were synthesized for use as positive MRI contrast agents with $T_{1}$-weighted sequences. ${ }^{24-27}$

\section{Study and optimization of the forced hydrolysis pathway in polyol}

The main reaction parameter to control IONP morphology (and therefore magnetic properties) was the solvent composition. A mixture of DEG and NMDEA (1:1 volume ratio) was used to yield large IONPs, while UUSPIO NPs were produced in pure DEG. In both cases, the same quantity and stoichiometric ratio of iron(II,III) chlorides and hydroxides were used $\left(\mathrm{Fe}^{3+} / \mathrm{Fe}^{2+} / \mathrm{OH}^{-} 2: 1: 8\right.$, i.e. one hydroxide anion per chloride). 
This document is the author manuscript version of published paper Inorganic Chemistry, 2017, 56(14), 8232-8243, after peer review and technical editing by the publisher. Final version visible on DOI:10.1021/acs.inorgchem.7b00956

a)

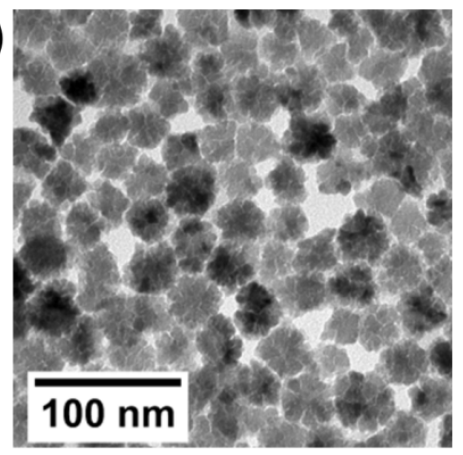

d)

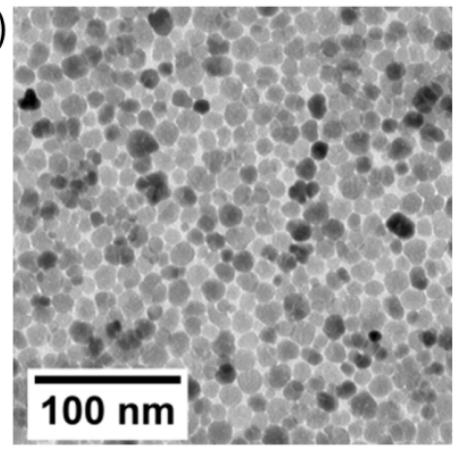

g)

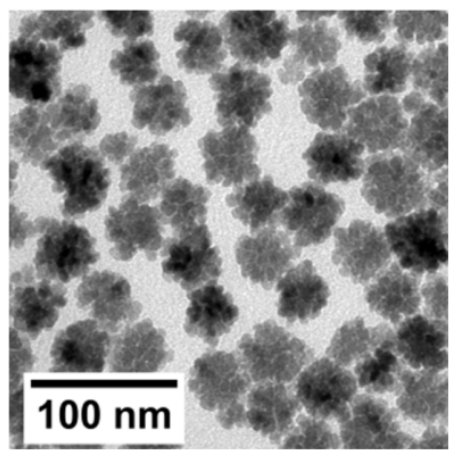

b)

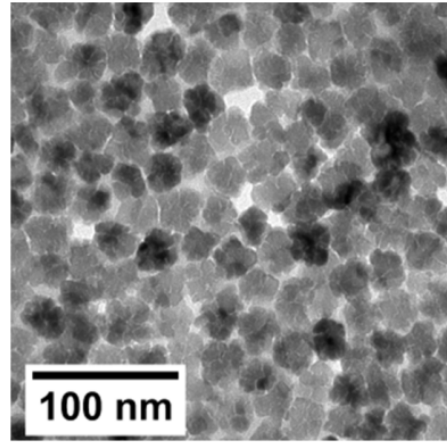

e)

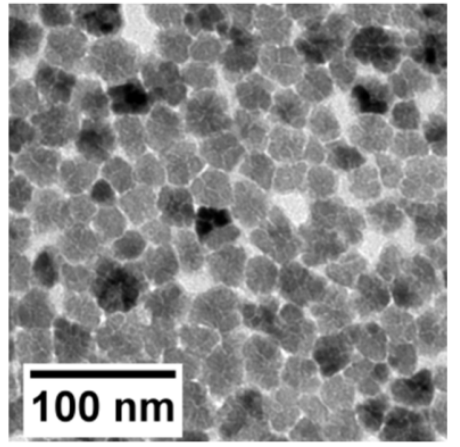

c)

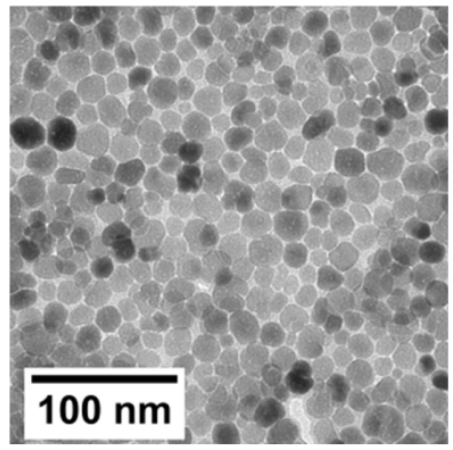

f)

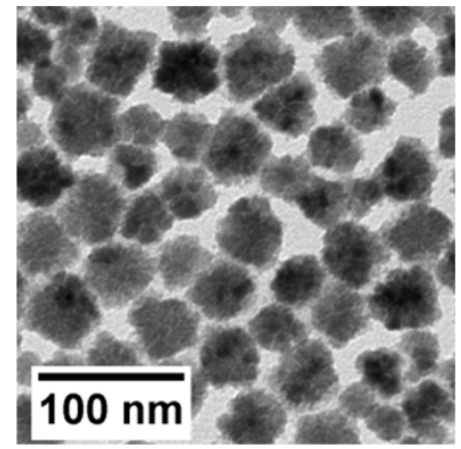

h)

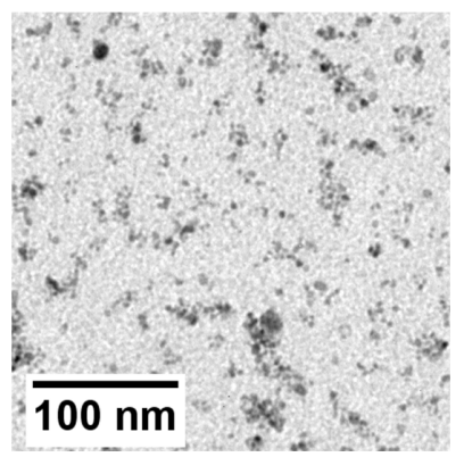

Figure 1. TEM micrographs of $\gamma-\mathrm{Fe}_{2} \mathrm{O}_{3}$ NPs of mono- or multi-core morphology: a) 36ff $(32.3 \pm 5.0 \mathrm{~nm})$, b) $35 \mathrm{ff}(29.1 \pm 4.4 \mathrm{~nm})$, c) $34 \mathrm{ff}(18.5 \pm 3.2 \mathrm{~nm})$, d) $32 \mathrm{ff}(14.5 \pm 3.4 \mathrm{~nm})$, e) $31 \mathrm{ff}$ $(27.5 \pm 4.2 \mathrm{~nm})$, f) $30 \mathrm{ff}(46.9 \pm 8.5 \mathrm{~nm}), \mathrm{g}) 15 \mathrm{ff}(36.9 \pm 4.8 \mathrm{~nm})$, and h) $17 \mathrm{ff}(4.3 \pm 1.1 \mathrm{~nm})$. TEM sizes in parentheses correspond to the mean outer diameters and respective standard deviations.

Reactants were heated from room temperature (RT) to reflux (approximately 30 min to reach $220{ }^{\circ} \mathrm{C}$ ) to obtain IONPs. Different quantities of water were injected to the reaction mixture either at RT before heating (heating-up, $H U$ ) or at reflux (hot injection, $H I$ ). Solvents used for the syntheses were freshly ordered and preserved from moisture. In the case of the 
This document is the author manuscript version of published paper Inorganic Chemistry, 2017, 56(14), 8232-8243, after peer review and technical editing by the publisher. Final version visible on DOI:10.1021/acs.inorgchem.7b00956

DEG/NMDEA solvent system, water was injected in different amounts, from $100 \mu \mathrm{L}$ to $2 \mathrm{~mL}$, into $120 \mathrm{~mL}$ of the total polyol solvent mixture, representing $0.083 \%$ to $1.67 \%$ volume ratios (v/v) or 5.5 to $110 \mathrm{mmol} \mathrm{H}_{2} \mathrm{O}$, i.e. stoichiometric (not catalytic) water quantity compared to the total iron(II,III) salts $(6 \mathrm{mmol})$. Lower water amounts did not allow producing IONPs, while larger quantities of water led to ill-defined IONPs. As seen on data (Figure 1 and Table 1), optimal control, in terms of size-distribution and morphology homogeneity, was achieved when mixing a determined amount of water in the solvent system with the precursors before heating $(H U)$, as supposedly ascribed to a more homogeneous composition of the starting mixture.

Surprisingly, using anhydrous iron(III) chloride instead of the hexahydrate compound did not allow producing magnetic IONPs, even when traces of water were added before heating $(H U)$. This evidences the critical role of water and its necessary presence in the starting iron salt precursors for the positive outcome of the reaction. In the polyol/poly(hydroxy)amine (DEG/NMDEA) synthesis indeed, the solvents act simultaneously as multivalent chelators for iron(II,III) cations, as well as a high boiling temperature medium to achieve a good control over the nucleation and separation from growth of IONPs. The chloride counter-ions of the iron(II,III) salts are thus exchanged by complexing solvent molecules and subsequently by hydroxide anions or by water molecules when adding the $\mathrm{NaOH}$ solution. It was described by Caruntu et al. that the actual precursors of inorganic polymerization are iron(II,III) hydroxides in which the metallic centers are also chelated by DEG. ${ }^{11}$ Therefore, the polyol route is also referred to in literature as a "forced hydrolysis" mechanism. This salt metathesis can be observed by eye when mixing the reactants from the color changes of the organometallic solution turning into a black colloidal suspension of magnetite $\left(\mathrm{Fe}_{3} \mathrm{O}_{4}\right)$ NPs. Studying the medium of synthesis by ${ }^{1} \mathrm{H}$ NMR spectroscopy helped understanding the mechanism of reaction (Figure S2). It was observed that 
This document is the author manuscript version of published paper Inorganic Chemistry, 2017, 56(14), 8232-8243, after peer review and technical editing by the publisher. Final version visible on DOI:10.1021/acs.inorgchem.7b00956

controlled water addition shifts the broad peak attributed to the labile protons of hydroxyl groups in DEG and NMDEA (at respectively 3.43 and $3.07 \mathrm{ppm}$ ). Apart from further shifting of this labile protons peak ascribed to $\mathrm{pH}$ variation occurring during the synthesis (hydroxyl groups being converted into oxides), the NMR spectrum does not show any evidence of polyol molecules degradation. It is worth noting that the IONPs are, in most cases, covered by a layer of chelating solvent molecules even after several washing steps using a mixture of ethanol and ethylacetate. $^{14}$ This affects the colloidal stability of the samples, and their ability to be oxidized by the boiling $\mathrm{Fe}\left(\mathrm{NO}_{3}\right)_{3}$ addition. ${ }^{17}$ During the oxidation step, the sample color is expected to turn from black to dark red as IONPs are oxidized from magnetite to maghemite. In some cases, especially for nanoflowers, the color of the colloidal solution remained black. This protective layer of DEG and NMDEA at the surface of IONPs was also evidenced by $\zeta$ potential measurement at varying $\mathrm{pH}$ (Figure S3). The isoelectric point (IEP) of maghemite is expected at around $\mathrm{pH}=7$, while the IEP of IONPs still covered by a layer of solvent ligands was shifted to about $\mathrm{pH}=9$, which is consistent with the expected $\mathrm{pKa}$ value of the tertiary amine in NMDEA. Washing IONPs by a precipitation-redispersion process in aqueous alkaline media revealed to be an efficient means to completely remove the remainder of chelated solvents as evidenced by the shift of the curve of $\zeta v s . \mathrm{pH}$ after extensive washing and by the ATR-IR spectrum on Figure S3.

\section{Structure and properties of the synthesized IONPs}

In the case of the sole DEG solvent, amounts of water as large as $5 \mathrm{~mL}$, representing $4.2 \%$ of the total solvent volume or $275 \mathrm{mmol} \mathrm{H}_{2} \mathrm{O}$ molecules, were injected at reflux temperature with a syringe needle through a septum (hot injection, $H I$ ), generating much smaller IONPs, with diameters typically in the order of 3-5 nm. The fast introduction of a water excess at high 
This document is the author manuscript version of published paper Inorganic Chemistry, 2017, 56(14), 8232-8243, after peer review and technical editing by the publisher. Final version visible on DOI:10.1021/acs.inorgchem.7b00956

temperature immediately generated a sudden nuclei burst, with a solution turning from deep green to black. This hot injection method lead to 'ultra-ultra-small' IONPs (UUSPIOs) as there is a limited quantity of precursors in solution available for crystal growth. The time-scale of reaction was usually much shorter than when synthesizing larger IONPs in DEG/NMDEA. Typically, reactions in sole DEG were completed after only 15-20 min. When letting an aliquot of the reaction mixture at rest over a strong permanent magnet, the supernatant became colorless, evidencing the total conversion of the colored iron(II,III) organometallic precursors into a colloidal magnetite phase.

The final products of all the different batches greatly differed in sizes and shapes according to the solvent composition: only DEG or DEG/NMDEA, or amount and timing of water addition. Smooth spheres as well as more complex structures previously reported as "nanoflowers" were obtained. TEM images enabled to determine both the overall diameters and the individual grain sizes for these multi-core IONPs. These estimates can be compared to other available techniques for particle sizing, namely the fit of the magnetization curve by the Langevin function convolved with a distribution law of diameters, or of the SANS curve by a polydisperse sphere form factor, these two methods being shown in supporting information (Figures S1 and S4). Smooth spheres were produced with adjustable sizes from $\sim 4 \mathrm{~nm}$ to $\sim 20 \mathrm{~nm}$ when the mixture was left at rest during all the reaction (under 'natural convection'), while nanoflowers were obtained when the mixture was continuously homogenized with a stirring shaft, with sizes from $\sim 27$ to $\sim 37 \mathrm{~nm}$ (Figure 1). The size-histograms could be well-fitted using a log-normal distribution law of parameters $\alpha$ and $\beta$ (Figure 2). In order to express diameters as $d_{0} \pm \sigma$, the mean values $d_{0}$ along with standard deviations $\sigma$ were calculated according to the following formulas:

$$
d_{0}=\langle d\rangle=\alpha e^{\beta^{2} / 2} \quad \text { and } \quad \sigma^{2}=\left\langle\left(d-d_{0}\right)^{2}\right\rangle=d_{0}^{2}\left(e^{\beta^{2}}-1\right)
$$


a)

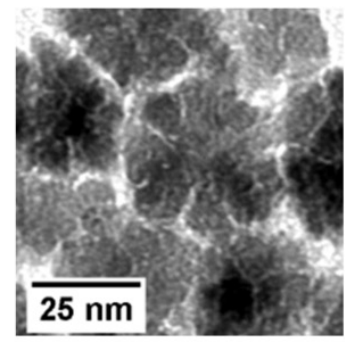

c)

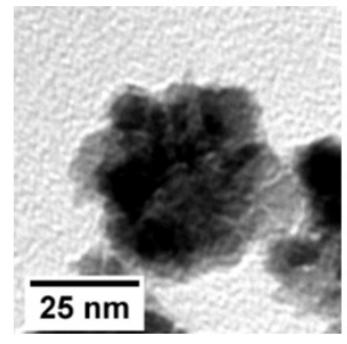

b)

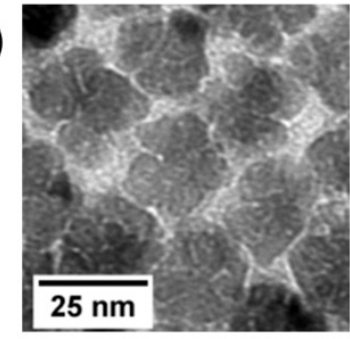

d)

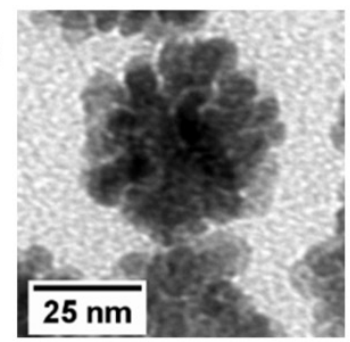

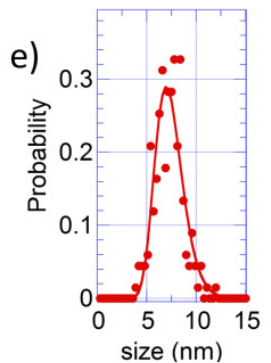

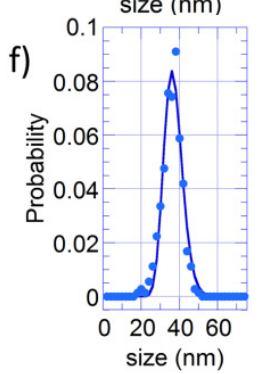

Figure 2. High magnification (HM) TEM micrographs of $\gamma-\mathrm{Fe}_{2} \mathrm{O}_{3}$ nanoflowers: a) 36ff, b) 35ff, c) $30 \mathrm{ff}$, and d) $15 \mathrm{ff}$. Distribution of e) grain sizes of the $15 \mathrm{ff}$ batch $(7.4 \pm 1.4 \mathrm{~nm})$, and $\mathrm{f}$ ) outer diameter $(36.9 \pm 4.8 \mathrm{~nm})$, measured on HM-TEM micrographs and log-normal fits.

Size distributions characterized by $\beta$ parameters below 0.2 were considered sufficiently narrow and suitable for further characterization. The condition $\mathrm{N} 00_{\mathrm{HU}^{-}} 5 \mathrm{~h}$, using mechanical stirring, proved to be both optimal and robust for the reproducible synthesis of nanoflowers as evidenced by the similar TEM images obtained for the $35 \mathrm{ff}$ and $36 \mathrm{ff}$ batches (Figure 2 ). In contrast, similar conditions without stirring $\left(\mathrm{N}_{100^{-}}-5 h^{*}\right)$ led to smooth spherical iron oxide NPs (34ff) of narrow size-distribution $18.5 \pm 3.2 \mathrm{~nm}$ as determined by TEM analysis, or $18.8 \pm 6.5 \mathrm{~nm}$ as obtained by fitting the DC magnetization curve (Figure S4). This observation highlights the critical role of mixing (diffusion - reaction $v$ s. active stirring) as experimental parameter on the resulting IONP morphology. 

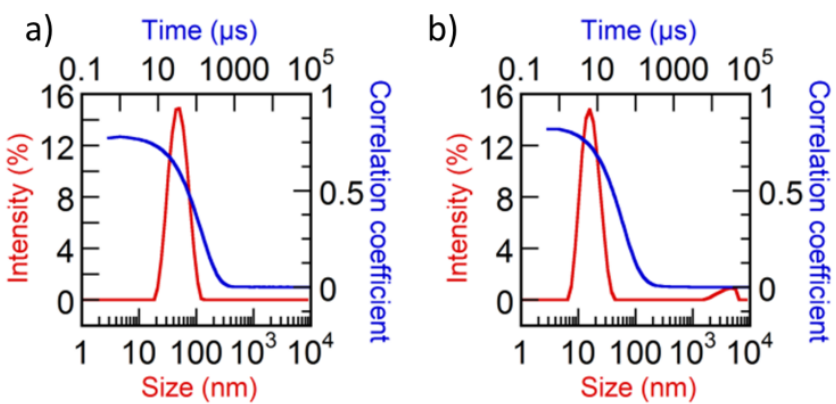

Figure 3. DLS correlograms and intensity-averaged distribution of diameters of a) $35 \mathrm{ff}$ nanoflowers $\left(D_{\mathrm{h}}=36 \mathrm{~nm}, \mathrm{PDI}=0.13\right)$, and b) 20ff UUSPIO smooth spheres $\left(D_{\mathrm{h}}=16 \mathrm{~nm}\right.$, $\mathrm{PDI}=0.21)$. Values in parentheses correspond to the $Z$-average hydrodynamic diameter $\left(D_{\mathrm{h}},\right)$ obtained from the $2^{\text {nd }}$ order cumulant fit, and polydispsersity index (PDI). See all DLS plots on

\section{Figure S5.}

On the other hand, the dispersion state of the different IONP batches synthesized was probed by dynamic light scattering (DLS) in a weakly acidic aqueous medium ( $\mathrm{pH} 2$ ). The fit of correlograms by the $2^{\text {nd }}$ order cumulant method provides the $Z$-average hydrodynamic diameter $\left(D_{\mathrm{h}}\right)$ and polydispersity index (PDI). Typical values of $Z$-average diameters were in the order of $30 \mathrm{~nm}$, ranging from 16 to $55 \mathrm{~nm}$ when considering all synthesized batches (Figure S5). For instance, the $Z$-average hydrodynamic diameter of $35 \mathrm{ff}$ nanoflowers was measured at $D_{\mathrm{h}}=36 \mathrm{~nm}$ $(\mathrm{PDI}=0.13)$, while 20ff UUSPIO smooth spheres were sized at $D_{\mathrm{h}}=16 \mathrm{~nm}(\mathrm{PDI}=0.21)$ (Figure 3). Hydrodynamic diameters estimated by DLS are always found larger than diameters determined by TEM due to the dried state of the TEM sample. The DLS sizes however represent better the real dispersion state of NPs. 
a)
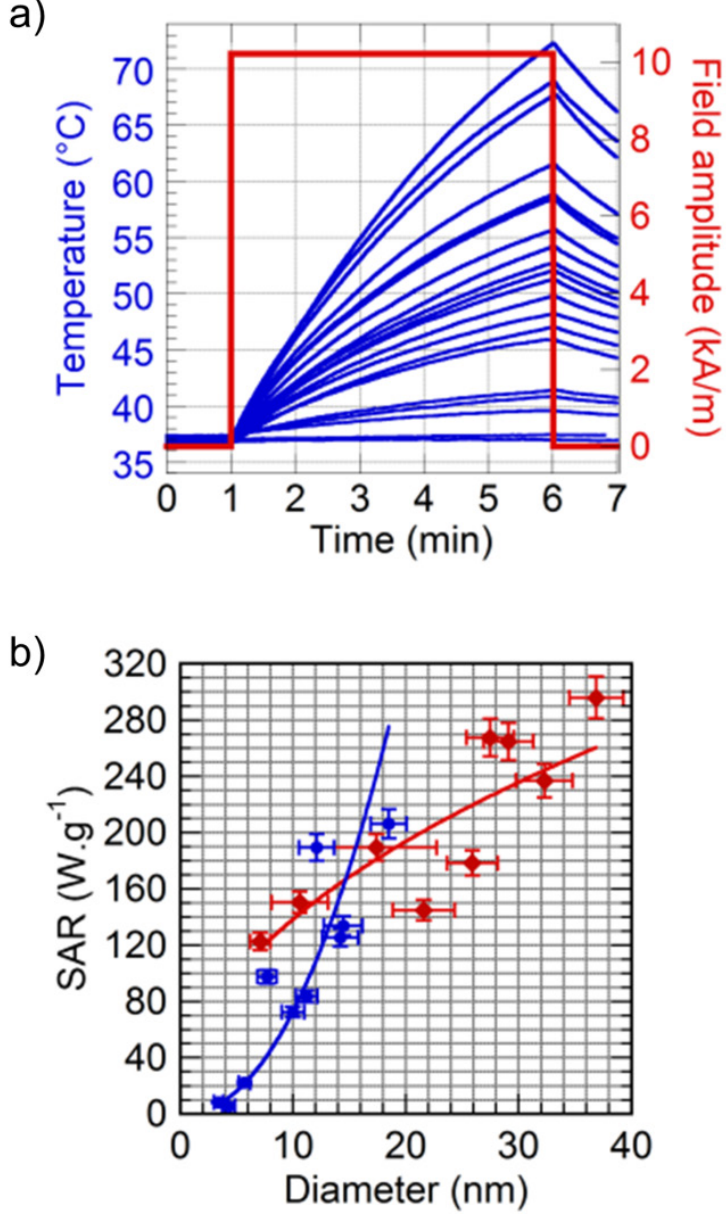

Figure 4. a) Temperature profiles vs. time of different samples of iron oxide concentration set at $3 \mathrm{~g} \cdot \mathrm{L}^{-1}$, under application of an AMF at $H_{\mathrm{app}}=10.2 \mathrm{kA} \cdot \mathrm{m}^{-1}$ and $f=755 \mathrm{kHz}$. The full list of samples and corresponding initial slopes (within first $5 \mathrm{~s}$ ) is given, on Table S1; b) Deduced SAR vs. mean diameter (determined by TEM) for smooth nanospheres (blue) and nanoflowers (red). Solid lines are power law fits of exponents 2.2 and 0.48 , respectively.

The efficiency of the different NP batches for magnetic hyperthermia was evaluated by applying an AMF for 5 min and by recording the temperature rise of the solvent, with iron oxide concentrations set to $3 \mathrm{~g} \cdot \mathrm{L}^{-1}$. While no significant heating under AMF was obtained for a few samples like the $17 \mathrm{ff}$ UUSPIOs with the smallest size, a temperature rise from $37{ }^{\circ} \mathrm{C}$ to $70{ }^{\circ} \mathrm{C}$ was recorded for the largest dispersible nanoflowers 15ff (Figure 4). Therapeutic hyperthermia 
This document is the author manuscript version of published paper Inorganic Chemistry, 2017, 56(14), 8232-8243, after peer review and technical editing by the publisher. Final version visible on DOI:10.1021/acs.inorgchem.7b00956

requires that IONPs heat cancerous tissues up to $43-44{ }^{\circ} \mathrm{C}$ to deposit a suitable "thermal dose", usually by IONP intratumoral injection. ${ }^{28}$ This temperature may potentially be reached in vivo with the best heating samples after few minutes, even at a concentration as low as the one used in our study $\left(3 \mathrm{~g} \cdot \mathrm{L}^{-1}\right)$, assuming that their heating properties are preserved in physiological intracellular conditions. ${ }^{29}$ The heating properties of our sample library were thus quantified using the specific absorption rate $(S A R)$ determined experimentally using the commonly used formula:

$$
S A R=(\Delta T / \Delta t)_{t \rightarrow 0} C_{P} / m
$$

where $(\Delta T / \Delta t)_{t \rightarrow 0}$ is the temperature raise slope at early times of AMF application (first $5 \mathrm{~s}$ ) to simulate adiabatic conditions, ${ }^{30} \mathrm{~m}$ is the mass of nanoparticles in $1 \mathrm{~mL}$ of suspension and $C_{P}$ is approximated by the specific heat of pure water. $^{2}$

We have used the $S A R$ to evaluate the heating properties of our nanoparticles instead of the intrinsic loss power $(I L P)^{31}$, because the $S A R$ variation with field intensity can depart significantly from a quadratic law. The plot of $S A R$ at given field amplitude $\left(H_{\mathrm{app}}=10.2 \mathrm{kA} \cdot \mathrm{m}^{-1}\right)$ and frequency $(f=755 \mathrm{kHz})$ vs. diameter measured by TEM clearly evidences a correlation between the $S A R$ and IONP outer diameter measured by TEM. (Figure 4b) Nanospheres experimentally follow a quadratic law with diameter while nanoflowers follow a lower exponent (nearly square-root). This is qualitatively in agreement with the most advanced models on the optimal size of magnetic NPs for MFH at given values of their other physical properties (specific magnetization and magnetic anisotropy). ${ }^{32}$ Results compiled in Table S1 evidence that all nanoflowers and smooth spheres larger than 12-14 nm are efficient nanoheaters, while UUSPIOs smaller than 5-6 nm do not generate sufficient heat but are likely useable as positive $\left(T_{1^{-}}\right.$ weighted) MRI contrast agents, as shown later in this article. Smooth spheres of intermediate diameters (10-14 nm) are ideal to be used both as nanoheaters for MFH and as negative $\left(T_{2^{-}}\right.$ 
This document is the author manuscript version of published paper Inorganic Chemistry, 2017, 56(14), 8232-8243, after peer review and technical editing by the publisher. Final version visible on DOI:10.1021/acs.inorgchem.7b00956

weighted) MRI contrast agents (vide infra), once coated with appropriate ligands, while larger ones might be more difficult to stabilise in physiological media.

An in-depth characterization of magnetic heating properties was carried out on selected IONP samples with interesting morphologies and core size in 20-40 $\mathrm{nm}$ range as evidenced by TEM, with outstanding heating efficiency $\left(S A R>200 \mathrm{~W} \cdot \mathrm{g}^{-1}\right.$ at $H_{\mathrm{app}}=10.2 \mathrm{kA} \cdot \mathrm{m}^{-1}$ and $\left.f=755 \mathrm{kHz}\right)$. Large smooth spheres (34ff) and nanoflowers of different grain size and increasing outer diameters (31ff, 35ff, and 15ff) were thus selected to be further examined with an in-house developed pickup coil set-up, allowing SAR measurement on a broad range of AMF frequencies and amplitudes. ${ }^{33}$ AC magnetization curves of NPs are plotted on Figure 5a versus amplitude $H_{\text {app }}$ up to $21 \mathrm{kA} . \mathrm{m}^{-1}$ at fixed frequency $(f=1030 \mathrm{kHz})$. Similar curves at different frequencies are provided as supporting information accompanying this manuscript (Figures S6, S7, S8 and S9).
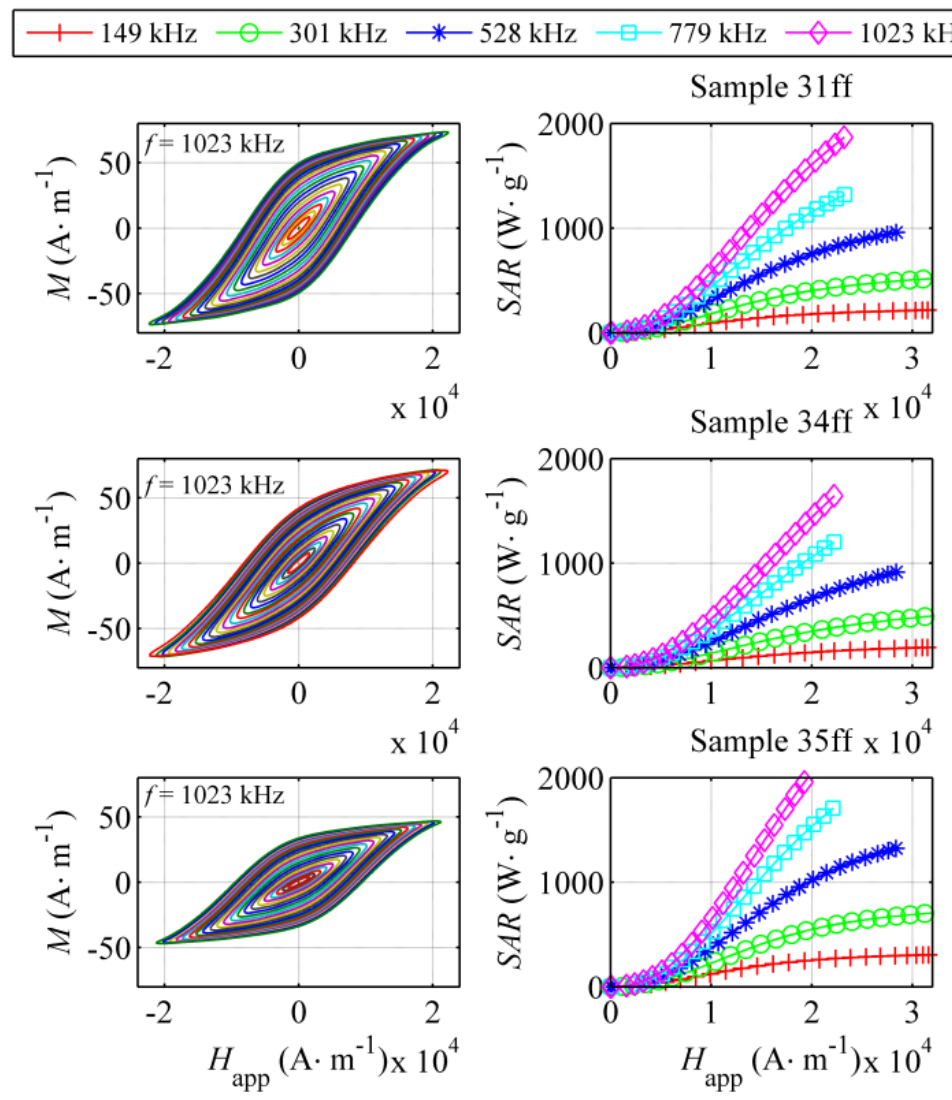
This document is the author manuscript version of published paper Inorganic Chemistry, 2017, 56(14), 8232-8243, after peer review and technical editing by the publisher. Final version visible on DOI:10.1021/acs.inorgchem.7b00956

Figure 5. a) AC hysteresis loops of samples $31 \mathrm{ff}, 34 \mathrm{ff}$ and $35 \mathrm{ff}$ at $1023 \mathrm{kHz}$ fixed frequency (left); b) SAR of samples $31 \mathrm{ff}, 34 \mathrm{ff}$ and $35 \mathrm{ff}$ versus field amplitude at different frequencies (right).

Such AC hysteresis loops reflect that magnetic moments of IONPs under AMF excitation oscillate with a phase lag relatively to the magnetic field, leading to a partial conversion of the radiofrequency magnetic energy into heat that then dissipates in the surrounding aqueous medium. Larger AC hysteresis loops areas are obtained at higher amplitudes $H_{\text {app }}$ and higher frequencies, while stronger magnetic anisotropy of the materials tends to change the shapes of the curves from sigmoidal to more square-like shapes. Carrey et al. interpreted such dynamic hysteresis loops by a two-level Stoner-Wohlfarth model instead of the classical linear response theory involving Néel and Brown relaxation times of the magnetic moments. ${ }^{32,34,35}$ The larger the surface of the hysteresis loops, the larger the energy dissipated by the IONPs per AMF cycle. These AC magnetization curves can be compared to the calorimetric experiments by multiplying the surface of the hysteresis loop with the frequency and by dividing by iron oxide concentration to get the $S A R$. The resulting values are then plotted as a function of frequency and amplitude, as shown in Figure 5b.
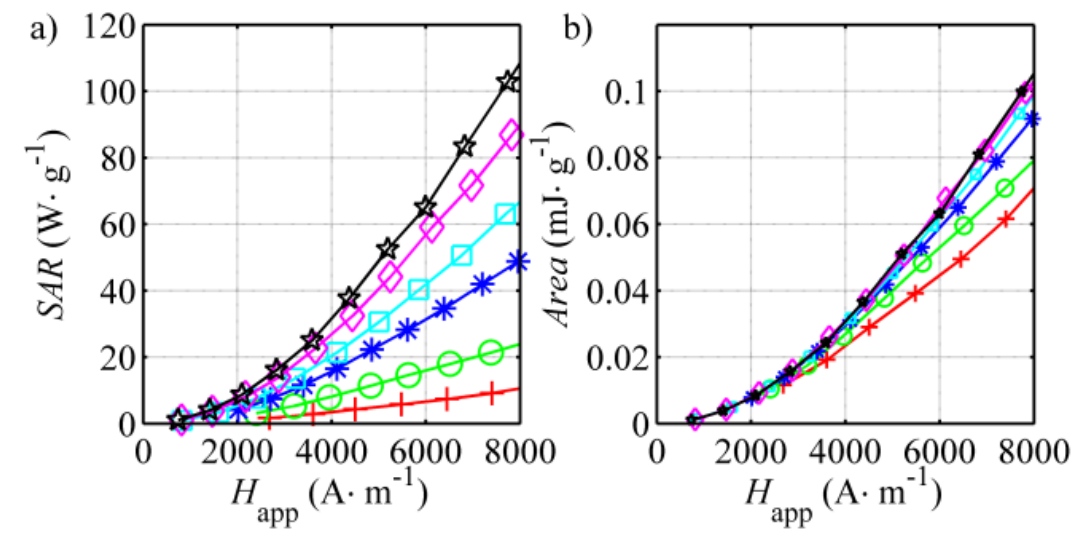
This document is the author manuscript version of published paper Inorganic Chemistry, 2017, 56(14), 8232-8243, after peer review and technical editing by the publisher. Final version visible on DOI:10.1021/acs.inorgchem.7b00956
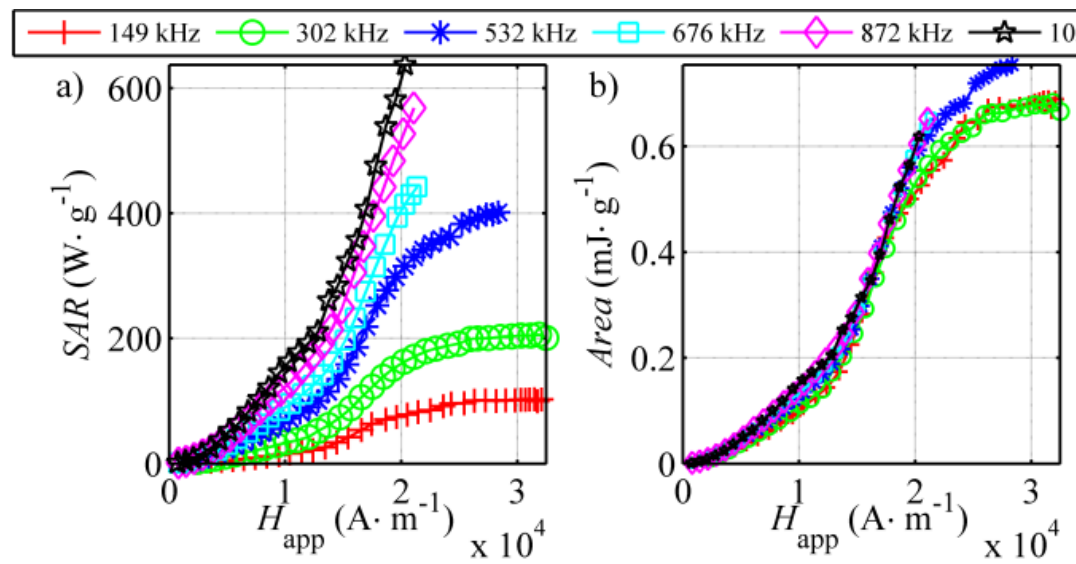

Figure 6. a) $S A R$ and b) hysteresis loop area $A v s$. applied intensity $H_{\text {app }}$ of the oxidized $15 \mathrm{ff}$ nanoflower batch. As can be appreciated on the zoomed curves at low $H_{\text {app }}$ (two upper graphs), the $15 \mathrm{ff}$ sample behaves as a superparamagnetic materials at low field ( $S A R$ varies as the square

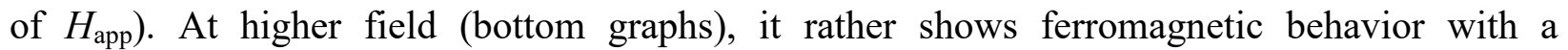
hysteresis area that first rises above threshold anisotropy field $H_{\text {an }}$ and then reaches a plateau.

$S A R$ values as high as $2000 \mathrm{~W} \cdot \mathrm{g}^{-1}$ were obtained at a frequency of $1023 \mathrm{kHz}$ and amplitude of $20 \mathrm{kA} \cdot \mathrm{m}^{-1}$, although out-passing by a factor 4 the upper limit of $5 \times 10^{9} \mathrm{~A} \cdot \mathrm{m}^{-1} \cdot \mathrm{s}^{-1}$ of the $f \cdot H_{\text {app }}$ product recommended for human treatment by $\mathrm{MFH} .^{36}$ Whereas the ILP parameter was previously introduced in the literature by dividing the $S A R$ with frequency and the square of $H_{\text {app }}{ }^{1}$ the assumption that $S A R$ is a quadratic function only works at low field amplitude (Figure 6a). We have therefore preferred to report the ratio (in $\mathrm{J}^{-1}{ }^{-1}$ ) of the $S A R$ and frequency, which represents the area $A$ of a hysteresis loop (i.e. thermal losses per AMF cycle). This enables to directly compare the heating properties of the magnetic IONPs synthesized in this work to different ones reported in literature, although measured under various conditions and setups. As reported by Carrey et al., ${ }^{32}$ the plot of hysteresis surface area $v s . H_{\text {app }}$ expected for ferromagnetic NPs exhibits a sigmoidal shape: it starts with a slow increase as long as the AMF amplitude is lower than the anisotropy field $H_{\mathrm{an}}$ of the nanomaterials, then increases rapidly (i.e. with an 
This document is the author manuscript version of published paper Inorganic Chemistry, 2017, 56(14), 8232-8243, after peer review and technical editing by the publisher. Final version visible on DOI:10.1021/acs.inorgchem.7b00956

exponent larger than two, value expected for superparamagnetic NPs), and finally plateaus in the high-field limit. The plot of $A$ vs. $H_{\text {app }}$ for the $15 \mathrm{ff}$ sample perfectly fits this description (Figure 6b), with a threshold anisotropy field $H_{\mathrm{an}} \sim 10^{4} \mathrm{~A} \cdot \mathrm{m}^{-1}$. The plateau value around $0.7 \mathrm{~mJ} \cdot \mathrm{g}^{-1}$ for the oxidized $15 \mathrm{ff}$ sample is not particularly outstanding as a maximal value of $1.8 \mathrm{~mJ} \cdot \mathrm{g}^{-1}$ was previously reported for IONPs obtained by coprecipitation followed by hydrothermal treatment. ${ }^{37}$ The oxidized $15 \mathrm{ff}$ sample however illustrates the complex magnetic behaviour of nanoflowers reflected in the dependence of their hysteresis loss area $A$ with the amplitude $H_{\text {app }}$ of the AMF: For the six probed frequencies, the plots of the hysteresis area $A$ vs. $H_{\text {app }}$ collapse almost perfectly on a unique master curve. The field dependence remains quadratic up to a threshold $H_{\text {app }}$ ascribed to the anisotropy field $H_{\text {an }}$ of the multi-core structure, characteristic of collective dynamics of sintered grains as in a multiple-domain magnet. For any applied AMF strength below $H_{\mathrm{an}}$, nanoflowers exhibit pure superparamagnetic response as evidenced by the quadratic variation of their $S A R$ vs. $H_{\text {app }}$ plot, each of their magnetic mono-domains being excited individually by the AMF. Other IONP batches of lower outer sizes (below $30 \mathrm{~nm}$ ) exhibit even superior plateau values of the hysteresis area per cycle $v s . H_{\text {app }}$ (which exact value slightly varies with frequency), from $\sim 1.6 \mathrm{~mJ} \cdot \mathrm{g}^{-1}$ for $31 \mathrm{ff}$ nanoflowers (Figure S10) and $34 \mathrm{ff}$ smooth nanospheres (Figure S11) to $\sim 2.5 \mathrm{~mJ} \cdot \mathrm{g}^{-1}$ for $35 \mathrm{ff}$ nanoflowers (Figure S12) or a bit lower (2 $\mathrm{mJ}^{-1} \mathrm{~g}^{-1}$ at the lowest frequency of $149 \mathrm{kHz}$. To the best of our knowledge, these are the highest hysteresis area values reported so far for synthetic magnetic IONPs, excluding the case of needle-like submicron $\gamma-\mathrm{Fe}_{2} \mathrm{O}_{3}$ particles commercialized for magnetic recording applications, that can reach hysteresis areas up to $8 \mathrm{~mJ} \cdot \mathrm{g}^{-1}$, but necessitate to apply an AMF stronger than a thrice higher threshold field, $H_{\mathrm{an}} \sim 30 \mathrm{kA} \cdot \mathrm{m}^{-1} \cdot{ }^{38}$ 
This document is the author manuscript version of published paper Inorganic Chemistry, 2017, 56(14), 8232-8243, after peer review and technical editing by the publisher. Final version visible on DOI:10.1021/acs.inorgchem.7b00956
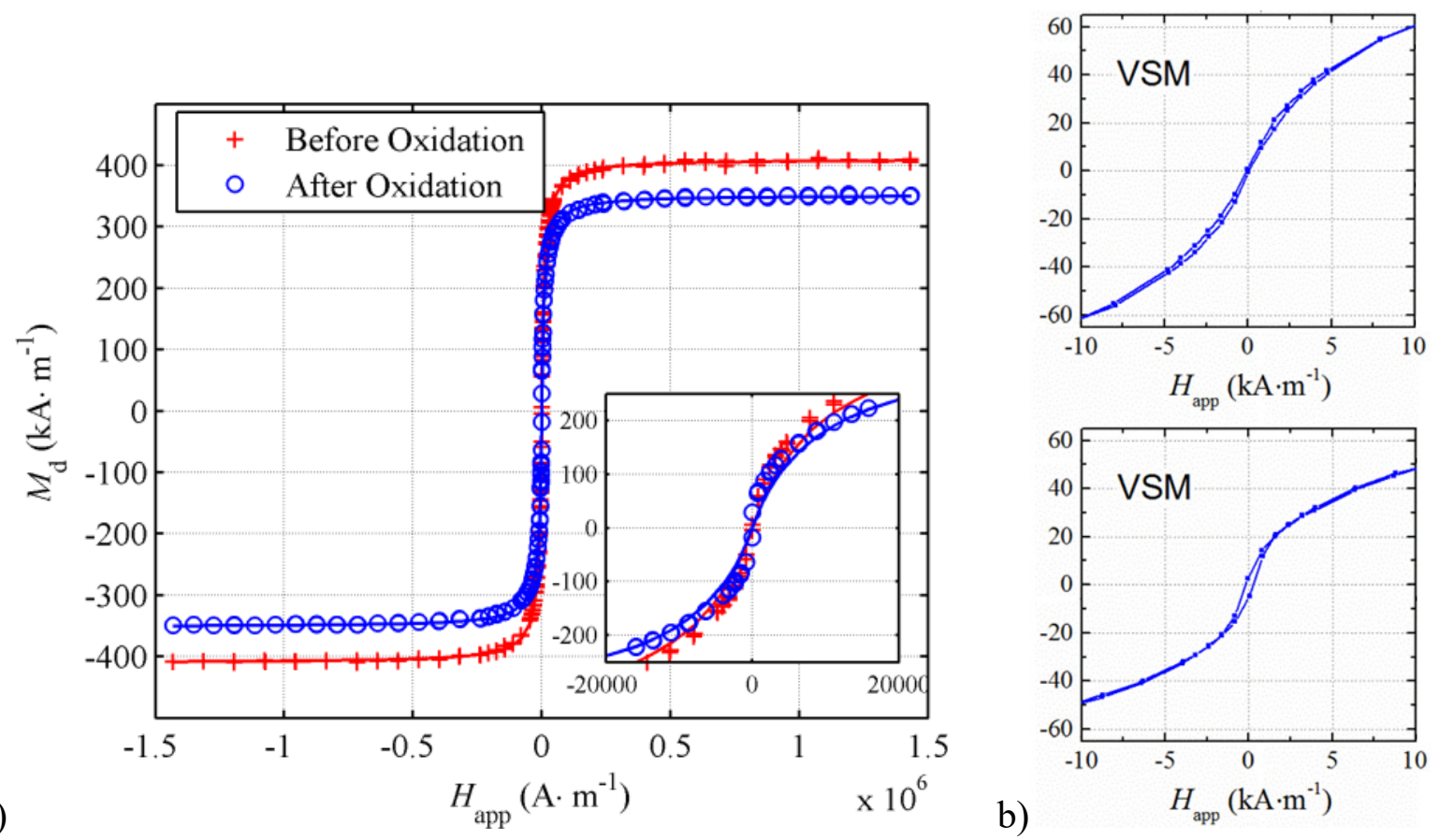

Figure 7. a) Static magnetization curve of $15 \mathrm{ff}$ nanoflowers measured by VSM at room temperature in water before and after oxidation (red and blue curves, respectively). In a narrow range $-1.6<H_{\mathrm{app}}<1.6 \mathrm{kA} \cdot \mathrm{m}^{-1}$ only, the curves can be approximated by $M_{\mathrm{d}}=\chi \cdot H_{\mathrm{app}}$, the susceptibility reaching a value as high as $\chi=59$ in both cases; b) Enlargements of the VSM curves in the low field region $\left(-10<H_{\mathrm{app}}<10 \mathrm{kA} \cdot \mathrm{m}^{-1}\right)$ of $15 \mathrm{ff}$ nanoflowers dispersed in an agar gel (upper curve) or in glycerol (lower curve), showing that the coercive field $\left(H_{\mathrm{c}}\right)$ and remnant magnetization $\left(M_{\mathrm{r}}\right)$ - if any - lay near the limit of sensitivity of the field probe i.e. $\sim 500 \mathrm{~A} \cdot \mathrm{m}^{-1}$.

To get an insight into the peculiar structure-property relationships and complex magnetic behavior of nanoflowers, the DC magnetization curves of $15 \mathrm{ff}$ IONPs (with or without the oxidation step) were also measured with a lab-made VSM setup (Figure 7). In both cases, the IONPs were dispersed in water acidified with dilute $\mathrm{HNO}_{3}(\mathrm{pH} 2.5)$. The magnetization curve of both samples exhibits null coercive field $\left(H_{\mathrm{c}}=0\right)$ and zero remanence $\left(M_{\mathrm{r}}=0\right)$, which corresponds to pure superparamagnetic behavior. This evidences the good dispersion state of these IONPs in 
This document is the author manuscript version of published paper Inorganic Chemistry, 2017, 56(14), 8232-8243, after peer review and technical editing by the publisher. Final version visible on DOI:10.1021/acs.inorgchem.7b00956

water, as a remnant magnetization would have been expected in case of an aggregated sample. The saturation magnetization of the non-oxidized sample obtained after synthesis and washings is $350 \mathrm{kA} \cdot \mathrm{m}^{-1}$, below the value of bulk magnetite $\left(\mathrm{Fe}_{3} \mathrm{O}_{4}\right)\left(500 \mathrm{kA} \cdot \mathrm{m}^{-1}\right)$, as ascribed to spincanting defects at the NP surface, ${ }^{39}$ or to partial oxidation already starting during the purification steps, as no particular precautions were taken to prevent it. In contrast, total oxidation of sample $15 \mathrm{ff}$ obtained by heating with iron(III) nitrate, led to a magnetization at saturation of $300 \mathrm{kA} \cdot \mathrm{m}^{-1}$, which is again below the expected value of $400 \mathrm{kA} \mathrm{m-1}$ for bulk maghemite $\left(\gamma-\mathrm{Fe}_{2} \mathrm{O}_{3}\right)$ but nevertheless quite a satisfying value for IONPs. Therefore, all the IONP batches were oxidized intentionally to control the magnetic phase of the IONPs, despite lowering the magnetic saturation and, presumably, their heating efficiency for MFH. All samples reported in this study exhibit similar saturation magnetization (Figure S4).

A second useful information provided by VSM magnetometry can be obtained by fitting the DC magnetization curves by the Langevin function characteristic of superparamagnetism, convolved by a log-normal distribution of diameters to take into account size-dispersity. ${ }^{21}$ The resulting magnetic domain diameters lay below the outer diameter measured by TEM for nanoflowers: $25.1 \pm 12.0 \mathrm{~nm}$ for $35 \mathrm{ff}, 21.9 \pm 10.6 \mathrm{~nm}$ for $31 \mathrm{ff}$, while almost identical to TEM (within experimental uncertainty) for $34 \mathrm{ff}$ smooth nanospheres: $18.8 \pm 6.5 \mathrm{~nm}$.

Sensitive magnetometry performed on the commercial MPMS system on the oxidized and non-oxidized 15ff nanoflowers (Figure S13) led to ZFC and FC magnetization curves vs. temperature both lower for the non-oxidized as compared to the oxidized $15 \mathrm{ff}$ NPs. Such nonclassical ZFC-FC curve profile has been already reported for large (18 and $22 \mathrm{~nm}) \mathrm{Fe}_{3} \mathrm{O}_{4} \mathrm{NPs}_{\mathrm{s}}$ synthesized by iron(III) oleate thermal decomposition, ${ }^{40}$ and was partially explained by the so- 
called Verwey or charge ordering transition, ${ }^{40,} 41$ when such IONPs undergo a slight crystallographic distortion from cubic, electrically conducting, to inverse spinel, electrically insulating, structure, this change of crystalline structure also impacting the magnetic properties. A suitable method to estimate the blocking temperature $T_{\mathrm{B}}$, defined at the transition from the ferrimagnetic state to the superparamagnetic regime, consists in plotting the derivative of the $M_{\mathrm{FC}}-M_{\mathrm{ZFC}}$ curve difference with respect to temperature. ${ }^{22}$ For the two different batches (nonoxidized and oxidized 15ff), the plot exhibits three maxima (Figure S13). The peak near $90 \mathrm{~K}$ is ascribed to the Verwey transition of magnetite, yet it is not clear why it also appears on the oxidized sample. The two other peaks correspond to characteristic temperatures, respectively near 200 and $300 \mathrm{~K}$. It is rather uncommon for a sample to exhibit two values of blocking temperatures. One hypothesis is that $T_{\mathrm{B} 1} \approx 200 \mathrm{~K}$ is ascribed to individual magnetic domains of diameter $7.4 \pm 1.4 \mathrm{~nm}$ and $T_{\mathrm{B} 2} \approx 300 \mathrm{~K}$ to the whole magnetic multi-core structure of outer diameter $36.9 \pm 4.8 \mathrm{~nm}$. The complete interpretation of these data, e.g. in term of superspin glass transition, would necessitate complementary AC susceptometry experiments $v s$. temperature, to study the slow relaxation dynamics of the frustrated spins in multi-core nanoflowers as reported by Kostopoulou et al., ${ }^{10}$ but it is beyond the scope of this article. 


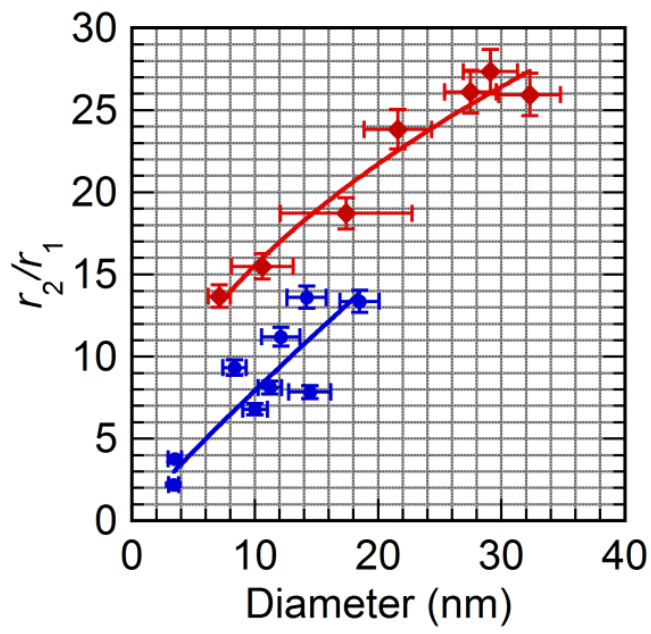

Figure 8. Ratio of transverse $\left(r_{2}\right)$ to longitudinal $\left(r_{1}\right)$ relaxivities at $1.41 \mathrm{~T} / 60 \mathrm{MHz}\left(37^{\circ} \mathrm{C}\right)$ vs. average TEM diameters of IONPs, for nanosphere (blue) and nanoflower (red) samples. Solid lines are power law fits of exponents respectively 0.92 and 0.48 .

In order to assess the efficiency of the different batches synthesized by the polyol route to relax nuclear spins of water protons, their transverse $\left(r_{2}\right)$ and longitudinal $\left(r_{1}\right)$ relaxivities were measured at physiological temperature $\left(37^{\circ} \mathrm{C}\right)$ with a $60 \mathrm{MHz}$ relaxometer based on a 1.41 Tesla magnet (i.e. close to the 1.5 Tesla magnetic field of most clinical MRI machines used in hospitals) (Figure 8). Practically, the longitudinal $\left(T_{1}\right)$ and transverse $\left(T_{2}\right)$ relaxation times of water protons were measured with an inversion-recovery and a CPMG sequence, respectively at decreasing equivalent $[\mathrm{Fe}]$ concentrations starting from $6 \mathrm{mM}$, in acidified water to maintain colloidal stability. Relaxivities are obtained from the slope of the linear variation with [Fe] of the longitudinal (respectively transverse) decay rate of water proton spins, according to: ${ }^{19}$

$$
1 / T_{i=1 \text { or } 2}=r_{i} \times[\mathrm{Fe}]+\left(1 / T_{i}\right)_{\mathrm{water}}
$$

where $\left(1 / T_{\mathrm{i}}\right)_{\text {water }}$ represents diamagnetic contribution of water.

In the "outer sphere" model of MRI contrast agents introduced by Ayant and Freed for paramagnetic agents, and adapted by Gillis et al. to superparamagnetic IONPs, ${ }^{42}$ the increase 
This document is the author manuscript version of published paper Inorganic Chemistry, 2017, 56(14), 8232-8243, after peer review and technical editing by the publisher. Final version visible on DOI:10.1021/acs.inorgchem.7b00956

relaxation rate $1 / T_{2}$ compared to pure water originates from fluctuating dipolar interactions between nuclear spins of water protons and the electronic magnetic moment of IONPs. For a limited range of diameters called "motional averaging regime", the superparamagnetic particle can be considered immobile during the echo time (TE) of the sequence compared to random trajectories of water molecules diffusing all around the magnetic sphere. In this case, Vuong et al. have shown that $r_{2}$ follows a universal scaling law that is quadratic both with the magnetization and with the radius of the "outer sphere", defined as the minimum approach distance between $\mathrm{H}_{2} \mathrm{O}$ molecules and the IONP center. ${ }^{43}$ For the IONP batches prepared in this study, the quadratic law is perfectly observed for the smooth sphere NPs (Figure S14), validating the proton diffusive model. This brings additional evidence that no organic layer remains on their surface after the washing steps. If not, water protons could not reach IONP surface and the quadratic law would not be observed. In the case of nanoflowers, the variation of $r_{2}$ vs. size appears erratic, presumably because of their rough geometry and very high specific area mentioned before, that can be up to $60 \%$ larger than the geometrical area of a smooth sphere (see case of ff35 batch on Figure S1). Proton relaxivity may arise by a combination of "outer sphere" and "inner sphere" mechanisms, meaning that water molecules can be transiently adsorbed in the porosity of nanoflowers, relaxation dynamics can therefore not be modelled by a single translational diffusion constant of water molecules around the particle.

The ratio of relaxivities $r_{2} / r_{1}$ is commonly used to determine whether NPs are most suitable as $T_{1}$ (positive) or $T_{2}$ (negative) MRI contrast agents. With $r_{2} / r_{1}$ ratios larger than 5 , most of the IONPs synthesized here are suitable as negative contrast agents for $T_{2}$ weighted MRI applications, such as commercial medical products Resovist ${ }^{\circledast}$, Feridex $^{\circledast}$, Cliavist $^{\circledR}$ or Clariscan $^{\circledR} .^{44}$ With much smaller $r_{2} / r_{1}$ ratios, UUSPIOs of just a few nm diameters synthesized in pure DEG 
This document is the author manuscript version of published paper Inorganic Chemistry, 2017, 56(14), 8232-8243, after peer review and technical editing by the publisher. Final version visible on DOI:10.1021/acs.inorgchem.7b00956

would rather be perfectly suitable as $T_{1}$-type, positive contrast agents. Such UUSPIO-based $T_{1^{-}}$ type contrast agents not yet commercially available would however be an excellent alternative to gadolinium chelates currently used and suspected of toxicity. ${ }^{45}$ Free gadolinium ions (i.e. not complexed by very strong ligands) are indeed nephrotoxic and neurotoxic, and undesirable side effects may arise in patients with renal insufficiency. Iron oxides having been proven perfectly safe in clinical use, these UUSPIOs are likely to find applications as alternative $T_{1}$ contrast agents in clinical MRI in a near future.

\section{CONCLUSIONS}

We have presented herein a comprehensive study of IONPs synthesis and characterization. IONPs were synthesized by the forced hydrolysis of iron(II, III) chlorides either in pure DEG polyol or in a mixture with NMDEA poly(hydroxy)amine. We have evidenced the importance of controlling the amount and timing of water addition for a successful synthesis and proper control over IONP morphology. A library of samples was obtained, ranging from "ultra-ultra-small” ( 3 $\mathrm{nm})$ UUSPIOs obtained by water "hot injection" in DEG at $220^{\circ} \mathrm{C}$ and fast growth $(20 \mathrm{~min})$, to large IONPs (up to $37 \mathrm{~nm}$ ) obtained by a longer "heating up" protocol in a DEG/NMDEA 1:1 mixture. Depending on reaction conditions (natural mixing vs. mechanical stirring), either smooth sphere or nanoflower morphologies were obtained. The structural and magnetic properties of these nanoparticles were extensively studied. They all exhibit superparamagnetic behavior characterized by a reversible magnetization curve in static magnetic field, with a strong saturation magnetization above $3 \times 10^{5} \mathrm{~A} \cdot \mathrm{m}^{-1}$. On the physical point of view, the specific absorption rate $(S A R)$ was first tested at given AMF conditions $\left(755 \mathrm{kHz}, 10.2 \mathrm{kA} \cdot \mathrm{m}^{-1}\right)$ and iron oxide concentration $\left(3 \mathrm{~g} \cdot \mathrm{L}^{-1}\right)$. These parameters were also varied over a broad range of field 
This document is the author manuscript version of published paper Inorganic Chemistry, 2017, 56(14), 8232-8243, after peer review and technical editing by the publisher. Final version visible on DOI:10.1021/acs.inorgchem.7b00956

strength and frequencies with the use of an $\mathrm{AC}$ magnetometer that is much faster than calorimetric experiments for estimating the SAR. The efficiency of most of synthesized batches as MRI contrast agents were also evaluated by proton relaxometry measurements. Several scaling laws were derived for the $S A R$ and the relaxivity ratio $r_{2} / r_{1}$, both being estimated at physiological body temperature $\left(37^{\circ} \mathrm{C}\right)$. At given $\mathrm{AMF}$ condition, the $S A R$ exhibits quadratic variation with diameter for smooth nanospheres and slower variations (viz. square root) for nanoflowers. Transverse relaxivity $r_{2}$ exhibits also quadratic variation with diameter for smooth nanospheres, in line with the "motional averaging regime" of the "outer sphere" model of MRI contrast agents. The $r_{2} / r_{1}$ ratios, calculated to distinguish IONPs better suited as $T_{1}$ or $T_{2}$ MRI contrast agents, varies linearly on diameter for nanospheres, and with a lower exponent (viz. square root) for nanoflowers. In the case of the smallest diameter IONPs synthesized (USPIOs), their longitudinal relaxivity $r_{1}$ at $1.41 \mathrm{~T}$ associated with moderate $r_{2} / r_{1}$ ratio make them alternatives to gadolinium chelates as positive MRI contrast agents, with lower risk of side effects on patients.

The AC hysteresis loops measured at varying AMF frequency and intensity brought more information on the magnetic hyperthermia mechanisms. In the case of nanoflowers and large nanospheres, the $S A R$ vs. field intensity curve shows an inflexion point between low and high fields. Below this threshold field, they present perfect superparamagnetic behavior, whereas above this field they behave more like ferromagnets. The threshold field can be associated with an anisotropy field as was done in the two-level Stoner-Wohlfarth model developed by Carrey et al. for blocked magnetic moments. ${ }^{32}$

Besides these obvious applications as nanoheaters in magnetic fluid hyperthermia and as MRI contrast agents, other uses can be envisioned. These very large diameter IONPs yet forming 
This document is the author manuscript version of published paper Inorganic Chemistry, 2017, 56(14), 8232-8243, after peer review and technical editing by the publisher. Final version visible on DOI:10.1021/acs.inorgchem.7b00956

stable colloidal suspensions exhibit extremely large magnetic susceptibility in DC magnetic field ( $\chi$ up to 100 ), which can be a requested property for delicate experimental setups based on weak DC magnetic fields such as the "magnetic tweezers" to manipulate living tissues or embryos, ${ }^{46}$ or micromechanical experiments to assess the flexural rigidity of magnetic wires. ${ }^{47}$

Another application could also be magnetic particle imaging (MPI) that requires IONPs optimally in the $20-25 \mathrm{~nm}$ diameter range so that magnetization saturates at low field strengths. ${ }^{48}$ Such versatility of sizes, morphologies and thus of physical properties was achieved by playing only on the nature of polyols as well as the amount and way of introducing water in the reaction vessel ("hot injection" vs. "heating up"), in solvent reflux conditions. In brief, robust, gram-scale and easily reproducible synthesis protocols were described to prepare from ultra-ultra-small superparamagnetic cores to very large size magnetic smooth nanospheres and nanoflowers, the latter offering among the highest magnetic heating properties reported so far for synthetic iron oxide nanoparticles.

\section{ASSOCIATED CONTENT}

Supporting Information. The Supporting Information is available free of charge on the ACS Publications website at DOI: 10.1021/acs.inorgchem.7b00956.

SANS curves, NMR analyses, Zeta potential, ATR-IR spectrum, DC magnetization curves, DLS curves, Summary of characteristics of the sample library, AC hysteresis cycles, SAR and hysteresis area vs. applied magnetic field at constant radiofrequency, ZFC-FC curves by MPMS magnetometry, longitudinal and transverse relaxivities $(\underline{\mathrm{PDF}})$. 
This document is the author manuscript version of published paper Inorganic Chemistry, 2017, 56(14), 8232-8243,

after peer review and technical editing by the publisher. Final version visible on DOI:10.1021/acs.inorgchem.7b00956

\section{AUTHOR INFORMATION}

\section{Corresponding Author}

* eneko.garayo@ehu.eus and olivier.sandre@enscbp.fr

\section{Author Contributions}

The manuscript was written through contributions of all authors. All authors have given approval to the final version of the manuscript.

\section{Funding Sources}

G.H.'s doctoral fellowship was funded by the 2014 call of the Department of Science and Technology of the University of Bordeaux (APUB1-ST2014). A. K.'s internship was supported by the US-France-Belgium iREU Site in Translational Chemistry funded by the National Science Foundation (Grant No. CHE 1560390) headed by Pr Randall J. Duran. Basque Government (Grant No. IT-1005-16) and Agence Nationale de la Recherche (Grant ANR-13-BS08-0017 MagnetoChemoBlast) are also acknowledged for financial support. This article is based upon work from COST Action RADIOMAG (TD1402), supported by COST (European Cooperation in Science and Technology).

\section{ACKNOWLEDGMENT}

Laboratoire Léon Brillouin (CEA-Saclay) also is acknowledged for giving access to G.H. to the PACE spectrometer during the 2015 annual training on neutron scattering techniques (Fan du LLB/Orphée). TEM images were taken at the Bordeaux Imaging Center (BIC) of the University of Bordeaux with the acknowledged help of Sabrina Lacomme and Etienne Gontier on equipment funded by France Life Imaging. Authors also want to thank SGIker (UPV/EHU) for the technical and human support as well as the VSM and ZFC-FC measurements. 
This document is the author manuscript version of published paper Inorganic Chemistry, 2017, 56(14), 8232-8243, after peer review and technical editing by the publisher. Final version visible on DOI:10.1021/acs.inorgchem.7b00956

\section{ABBREVIATIONS}

AMF, alternating magnetic field; DEG, diethylene glycol; DLS, dynamic light scattering; ATR-IR, attenuated total reflection infrared spectroscopy; ILP, intrinsic loss power; IONP, iron oxide nanoparticle; IEP, isoelectric point; MFH, magnetic fluid hyperthermia; MPMS, magnetic property measurement system; NMDEA, $N$-methyl diethanolamine; NP, nanoparticle; OD, optical density (or absorbance); SANS, small angle neutron scattering; $S A R$, specific absorption rate; UUSPIO, ultra-ultra-small superparamagnetic iron oxide.

\section{REFERENCES}

1. Hervault, A.; Thanh, N. T. K. Magnetic nanoparticle-based therapeutic agents for thermo-chemotherapy treatment of cancer. Nanoscale 2014, 6 (20), 11553-11573.

2. Périgo, E. A.; Hemery, G.; Sandre, O.; Ortega, D.; Garaio, E.; Plazaola, F.; Teran, F. J. Fundamentals and advances in magnetic hyperthermia. Applied Physics Reviews 2015, 2 (4), 041302.

3. Massart, R. Preparation of aqueous magnetic liquids in alkaline and acidic media. IEEE Transactions on Magnetics 1981, 17 (2), 1247-1248.

4. Caruntu, D.; Caruntu, G.; Chen, Y.; O'Connor, C. J.; Goloverda, G.; Kolesnichenko, V. L. Synthesis of Variable-Sized Nanocrystals of Fe3O4 with High Surface Reactivity. Chemistry of Materials 2004, 16 (25), 5527-5534.

5. Hyeon, T.; Lee, S. S.; Park, J.; Chung, Y.; Na, H. B. Synthesis of Highly Crystalline and Monodisperse Maghemite Nanocrystallites without a Size-Selection Process. J. Am. Chem. Soc. 2001, 123 (51), 12798-12801.

6. Horner, O.; Neveu, S.; Montredon, S. d.; Siaugue, J.-M.; Cabuil, V. Hydrothermal synthesis of large maghemite nanoparticles: influence of the $\mathrm{pH}$ on the particle size. J. Nanopart. Res. 2009, 11 (5), 1247-1250.

7. Shubitidze, F.; Kekalo, K.; Stigliano, R.; Baker, I. Magnetic nanoparticles with high specific absorption rate of electromagnetic energy at low field strength for hyperthermia therapy. Journal of Applied Physics 2015, 117 (9), 094302.

8. Blanco-Andujar, C.; Ortega, D.; Southern, P.; Pankhurst, Q. A.; Thanh, N. T. K. High performance multi-core iron oxide nanoparticles for magnetic hyperthermia: microwave synthesis, and the role of core-to-core interactions. Nanoscale 2015, 7 (5), 1768-1775.

9. Lartigue, L.; Hugounenq, P.; Alloyeau, D.; Clarke, S. P.; Lévy, M.; Bacri, J.-C.; Bazzi, R.; Brougham, D. F.; Wilhelm, C.; Gazeau, F. Cooperative Organization in Iron Oxide MultiCore Nanoparticles Potentiates Their Efficiency as Heating Mediators and MRI Contrast Agents. ACS Nano 2012, 6 (12), 10935-10949. 
This document is the author manuscript version of published paper Inorganic Chemistry, 2017, 56(14), 8232-8243, after peer review and technical editing by the publisher. Final version visible on DOI:10.1021/acs.inorgchem.7b00956

10. Kostopoulou, A.; Brintakis, K.; Vasilakaki, M.; Trohidou, K. N.; Douvalis, A. P.; Lascialfari, A.; Manna, L.; Lappas, A. Assembly-mediated interplay of dipolar interactions and surface spin disorder in colloidal maghemite nanoclusters. Nanoscale 2014, 6 (7), 3764-3776.

11. Caruntu, D.; Remond, Y.; Chou, N. H.; Jun, M.-J.; Caruntu, G.; He, J.; Goloverda, G.; O'Connor, C.; Kolesnichenko, V. Reactivity of 3d Transition Metal Cations in Diethylene Glycol Solutions. Synthesis of Transition Metal Ferrites with the Structure of Discrete Nanoparticles Complexed with Long-Chain Carboxylate Anions. Inorganic Chemistry 2002, 41 (23), 61376146.

12. Cheng, C.; Xua, F.; Gu, H. Facile synthesis and morphology evolution of magnetic iron oxide nanoparticles in different polyol processes. NewJ. Chem. 2011, 35, 1072-1079.

13. Hachani, R.; Lowdell, M.; Birchall, M.; Hervault, A.; Mertz, D.; Begin-Colin, S.; Thanh, N. T. K. Polyol synthesis, functionalisation, and biocompatibility studies of superparamagnetic iron oxide nanoparticles as potential MRI contrast agents. Nanoscale 2016, 8 (6), 3278-3287.

14. Hugounenq, P.; Levy, M.; Alloyeau, D.; Lartigue, L.; Dubois, E.; Cabuil, V.; Ricolleau, C.; Roux, S.; Wilhelm, C.; Gazeau, F.; Bazzi, R. Iron Oxide Monocrystalline Nanoflowers for Highly Efficient Magnetic Hyperthermia. J. Phys. Chem. C 2012, 116 (29), 15702-15712.

15. Forge, D.; Roch, A.; Laurent, S.; Tellez, H.; Gossuin, Y.; Renaux, F.; Vander Elst, L.; Muller, R. N. Optimization of the Synthesis of Superparamagnetic Contrast Agents by the Design of Experiments Method. The Journal of Physical Chemistry C 2008, 112 (49), 1917819185.

16. Sakellari, D.; Brintakis, K.; Kostopoulou, A.; Myrovali, E.; Simeonidis, K.; Lappas, A.; Angelakeris, M. Ferrimagnetic nanocrystal assemblies as versatile magnetic particle hyperthermia mediators. Materials Science and Engineering: C 2016, 58, 187-193.

17. Tourinho, F. A.; Franck, R.; Massart, R. Aqueous ferrofluids based on manganese and cobalt ferrites. Journal of Materials Science 1990, 25 (7), 3249-3254.

18. Koppel, D. E. Analysis of Macromolecular Polydispersity in Intensity Correlation Spectroscopy: The Method of Cumulants. The Journal of Chemical Physics 1972, 57 (11), 48144820.

19. Henoumont, C.; Laurent, S.; Vander Elst, L. How to perform accurate and reliable measurements of longitudinal and transverse relaxation times of MRI contrast media in aqueous solutions. Contrast Media \& Molecular Imaging 2009, 4 (6), 312-321.

20. Hemery, G.; Garanger, E.; Lecommandoux, S.; Wong, A. D.; Gillies, E. R.; Pedrono, B.; Bayle, T.; Jacob, D.; Sandre, O. Thermosensitive polymer-grafted iron oxide nanoparticles studied by in situ dynamic light backscattering under magnetic hyperthermia. Journal of Physics D: Applied Physics 2015, 48 (49), 494001.

21. Garaio, E.; Sandre, O.; Collantes, J.-M.; Garcia, J. A.; Mornet, S.; Plazaola, F. Specific absorption rate dependence on temperature in magnetic field hyperthermia measured by dynamic hysteresis losses (ac magnetometry). Nanotechnology 2015, 26 (1), 015704.

22. Bruvera, I. J.; Mendoza Zélis, P.; Pilar Calatayud, M.; Goya, G. F.; Sánchez, F. H. Determination of the blocking temperature of magnetic nanoparticles: The good, the bad, and the ugly. Journal of Applied Physics 2015, 118 (18), 184304.

23. Brûlet, A.; Lairez, D.; Lapp, A.; Cotton, J.-P. Improvement of data treatment in smallangle neutron scattering. Journal of Applied Crystallography 2007, 40, 165-177.

24. Tromsdorf, U. I.; Bruns, O. T.; Salmen, S. C.; Beisiegel, U.; Weller, H. A Highly Effective, Nontoxic T1 MR Contrast Agent Based on Ultrasmall PEGylated Iron Oxide Nanoparticles. Nano Letters 2009, 9 (12), 4434-4440. 
This document is the author manuscript version of published paper Inorganic Chemistry, 2017, 56(14), 8232-8243, after peer review and technical editing by the publisher. Final version visible on DOI:10.1021/acs.inorgchem.7b00956

25. Ninjbadgar, T.; Brougham, D. F. Epoxy Ring Opening Phase Transfer as a General Route to Water Dispersible Superparamagnetic Fe3O4 Nanoparticles and Their Application as Positive MRI Contrast Agents. Advanced Functional Materials 2011, 21 (24), 4769-4775.

26. Borase, T.; Ninjbadgar, T.; Kapetanakis, A.; Roche, S.; O'Connor, R.; Kerskens, C.; Heise, A.; Brougham, D. F. Stable Aqueous Dispersions of Glycopeptide-Grafted Selectably Functionalized Magnetic Nanoparticles. Angewandte Chemie International Edition 2013, 52 (11), 3164-3167.

27. Hannecart, A.; Stanicki, D.; Vander Elst, L.; Muller, R. N.; Lecommandoux, S.; Thevenot, J.; Bonduelle, C.; Trotier, A.; Massot, P.; Miraux, S.; Sandre, O.; Laurent, S. Nanothermometers with thermo-sensitive polymer grafted USPIOs behaving as positive contrast agents in low-field MRI. Nanoscale 2015, 7 (8), 3754-3767.

28. Sandre, O.; Genevois, C.; Garaio, E.; Adumeau, L.; Mornet, S.; Couillaud, F. In Vivo Imaging of Local Gene Expression Induced by Magnetic Hyperthermia. Genes 2017, 8 (2), 61.

29. Sanz, B.; Calatayud, M. P.; De Biasi, E.; Lima, E., Jr.; Mansilla, M. V.; Zysler, R. D.; Ibarra, M. R.; Goya, G. F. In Silico before In Vivo: how to Predict the Heating Efficiency of Magnetic Nanoparticles within the Intracellular Space. Sci Rep. 2016, 6, 38733.

30. Iacob, N.; Schinteie, G.; Palade, P.; Ticos, C. M.; Kuncser, V. Stepped heating procedure for experimental SAR evaluation of ferrofluids. The European Physical Journal E 2015, 38 (6), 57.

31. Kallumadil, M.; Tada, M.; Nakagawa, T.; Abe, M.; Southern, P.; Pankhurst, Q. A. Suitability of commercial colloids for magnetic hyperthermia. Journal of Magnetism and Magnetic Materials 2009, 321 (10), 1509-1513.

32. Carrey, J.; Mehdaoui, B.; Respaud, M. Simple models for dynamic hysteresis loop calculations of magnetic single-domain nanoparticles: Application to magnetic hyperthermia optimization. Journal of Applied Physics 2011, 109 (8), 083921.

33. Garaio, E.; Collantes, J. M.; Garcia, J. A.; Plazaola, F.; Mornet, S.; Couillaud, F.; Sandre, $\mathrm{O}$. A wide-frequency range $\mathrm{AC}$ magnetometer to measure the specific absorption rate in nanoparticles for magnetic hyperthermia. Journal of Magnetism and Magnetic Materials 2014, $368,432-437$.

34. Lacroix, L.-M.; Malaki, R. B.; Carrey, J.; Lachaize, S.; Respaud, M.; Goya, G. F.; Chaudret, B. Magnetic hyperthermia in single-domain monodisperse FeCo nanoparticles: Evidences for Stoner-Wohlfarth behavior and large losses. Journal of Applied Physics 2009, 105 (2), 023911.

35. Mehdaoui, B.; Meffre, A.; Carrey, J.; Lachaize, S.; Lacroix, L.-M.; Gougeon, M.; Chaudret, B.; Respaud, M. Optimal Size of Nanoparticles for Magnetic Hyperthermia: A Combined Theoretical and Experimental Study. Advanced Functional Materials 2011, 21 (23), 4573-4581.

36. Hergt, R.; Dutz, S. Magnetic particle hyperthermia-biophysical limitations of a visionary tumour therapy. Journal of Magnetism and Magnetic Materials 2007, 311 (1), 187192.

37. Marciello, M.; Connord, V.; Veintemillas-Verdaguer, S.; Verges, M. A.; Carrey, J.; Respaud, M.; Serna, C. J.; Morales, M. P. Large scale production of biocompatible magnetite nanocrystals with high saturation magnetization values through green aqueous synthesis. Journal of Materials Chemistry B 2013, 1 (43), 5995-6004. 
This document is the author manuscript version of published paper Inorganic Chemistry, 2017, 56(14), 8232-8243, after peer review and technical editing by the publisher. Final version visible on DOI:10.1021/acs.inorgchem.7b00956

38. Kashevsky, B. E.; Kashevsky, S. B.; Korenkov, V. S.; Istomin, Y. P.; Terpinskaya, T. I.; Ulashchik, V. S. Magnetic hyperthermia with hard-magnetic nanoparticles. Journal of Magnetism and Magnetic Materials 2015, 380, 335-340.

39. Baaziz, W.; Pichon, B. P.; Fleutot, S.; Liu, Y.; Lefevre, C.; Greneche, J.-M.; Toumi, M.; Mhiri, T.; Begin-Colin, S. Magnetic Iron Oxide Nanoparticles: Reproducible Tuning of the Size and Nanosized-Dependent Composition, Defects, and Spin Canting. The Journal of Physical Chemistry C 2014, 118 (7), 3795-3810.

40. Salas, G.; Casado, C.; Teran, F. J.; Miranda, R.; Serna, C. J.; Morales, M. P. Controlled synthesis of uniform magnetite nanocrystals with high-quality properties for biomedical applications. Journal of Materials Chemistry 2012, 22 (39), 21065-21075.

41. Goya, G. F.; Berquó, T. S.; Fonseca, F. C.; Morales, M. P. Static and dynamic magnetic properties of spherical magnetite nanoparticles. Journal of Applied Physics 2003, 94 (5), 35203528.

42. Gillis, P.; Roch, A.; Brooks, R. A. Corrected Equations for Susceptibility-Induced T2Shortening. Journal of Magnetic Resonance 1999, 137 (2), 402-407.

43. Vuong, Q. L.; Berret, J.-F.; Fresnais, J.; Gossuin, Y.; Sandre, O. A Universal Scaling Law to Predict the Efficiency of Magnetic Nanoparticles as MRI T2-Contrast Agents. Advanced Healthcare Materials 2012, 1 (4), 502-512.

44. Wang, Y.-X. J. Superparamagnetic iron oxide based MRI contrast agents: Current status of clinical application. Quantitative Imaging in Medicine and Surgery 2011, 1 (1), 35-40.

45. Thomsen, H. S.; Morcos, S. K.; Dawson, P. Is there a causal relation between the administration of gadolinium based contrast media and the development of nephrogenic systemic fibrosis (NSF)? Clinical Radiology 2006, 61 (11), 905-906.

46. Serwane, F.; Mongera, A.; Rowghanian, P.; Kealhofer, D. A.; Lucio, A. A.; Hockenbery, Z. M.; Campas, O. In vivo quantification of spatially varying mechanical properties in developing tissues. Nat Meth 2017, 14 (2), 181-186.

47. Gerbal, F.; Wang, Y. Optical detection of nanometric thermal fluctuations to measure the stiffness of rigid superparamagnetic microrods. Proceedings of the National Academy of Sciences 2017, 114 (10), 2456-2461.

48. Bauer, L. M.; Situ, S. F.; Griswold, M. A.; Samia, A. C. S. High-performance iron oxide nanoparticles for magnetic particle imaging - guided hyperthermia (hMPI). Nanoscale 2016, 8 (24), 12162-12169. 
This document is the author manuscript version of published paper Inorganic Chemistry, 2017, 56(14), 8232-8243, after peer review and technical editing by the publisher. Final version visible on DOI:10.1021/acs.inorgchem.7b00956

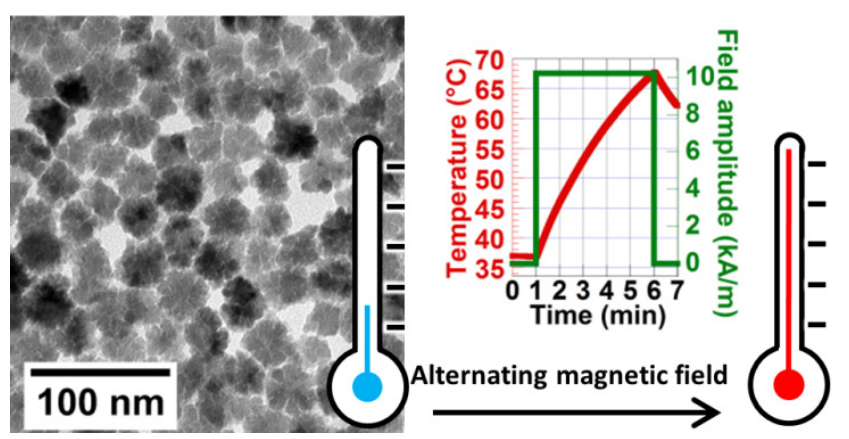

For Table of Contents Only. The polyol route offers an excellent compromise between size and shape control and industrial up-scalability for the synthesis of iron oxide nanoparticles. However, this study highlights the critical role played by stoichiometric amounts of water during the forced hydrolysis mechanism, and describes a full library of samples of varying structures (mono-core vs. multi-core) and properties optimized for magnetic fluid hyperthermia and/or MRI contrast. 